\title{
CMOS-MEMS Resonant Pressure Sensors: Optimization and Validation through Comparative Analysis
}

\author{
Saoni Banerji • Piotr Michalik • Daniel Fernández • Jordi Madrenas • Albert Mola • Josep Montanyà
}

\begin{abstract}
An optimized CMOS-MEMS resonant pressure sensor with enhanced sensitivity at atmospheric pressure has been reported in this paper. The presented work reports modeling and characterization of a resonant pressure sensor, based on the variation of the quality factor with pressure. The relevant regimes of air flow have been determined by the Knudsen number $\left(K_{n}\right)$, which is the ratio of the mean free path of the gas molecule to the characteristic length of the device. The sensitivity has been monitored for the resonator design from low vacuum to atmospheric levels of air pressure. This has been accomplished by reducing the characteristic length and optimization of other parameters for the device. While the existing analytical model has been adapted to simulate the squeeze film damping effectively and it is validated at higher values of air pressure, it fails to compute the structural damping mechanisms dominant in the molecular flow regime, i.e. at lower levels of air pressure. This discrepancy has been solved by finite element modeling that has incorporated both structural and film damping effects. The sensor has been designed with an optimal geometry of $140 \mu \mathrm{m} \times 140 \mu \mathrm{m} \times 8 \mu \mathrm{m}$ having $6 \times 6$ perforations along the row and column of the plate respectively, for maximum $Q$, with an effective mass of $0.4 \mu \mathrm{g}$. An enhanced quality factor of 60 and reduced damping coefficient of $4.34 \mu \mathrm{Ns} / \mathrm{m}$ have been obtained for the reported device at atmospheric pressure. The sensitivity of the manufactured device is approximately -0.09 at atmospheric pressure and increases to -0.3 at $40 \mathrm{kPa}$ i.e. in the lower pressures of slip flow regime. The experimental measurements of the manufactured resonant pressure sensor have been compared with that of the analytical and finite element modeling to validate the optimization procedure. The device has been manufactured using standard 250-nm CMOS technology followed by an in-house BEOL metal-layer release through wet etching.
\end{abstract}

Keywords: CMOS-MEMS resonant pressure sensor, squeeze film damping, quality factor, Knudsen number, perforated plate, optimization

\section{Introduction}

In recent years, monolithically integrated CMOS-MEMS resonant pressure sensors have been extensively used in the fields of atmospheric pressure monitoring and altitude sensing due to their advantages of low cost, small size and high reliability (Baltes 2005; Chen 2011). Contrary to their counterparts' i.e. capacitive, piezoresistive and piezoelectric pressure sensors, resonant pressure sensors provide quasi-digital output i.e. they allow direct coupling to digital electronics without the requirement of analog to digital converters (ADCs). This feature enhances their resolution and reliability by providing more immunity to noise and interference (Yinan 2015). Due to the large surface area to volume ratio, air damping plays a significant role in determining the net damping coefficient in micro-transducers (Bao 2003). Squeeze film damping is the dominant damping mechanism in the viscous flow regime of pressure. Other pressure independent sources of damping such as thermoelastic damping and structural damping (anchor losses, intrinsic losses) are negligible at atmospheric pressure. The squeeze-film damping effect arises when the fluid between two

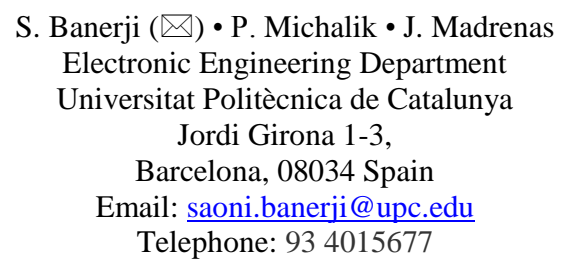

\section{Fernández • J. Montanyà} Nanusens

Av. del Parc Tecnològic, 3 CENT - Parc Tecnològic del Vallès 08290 Cerdanyola del Vallès, Spain

\author{
A. Mola-Romero \\ InContext $\mathrm{AB}$ \\ LM Ericssons väg 23 \\ 12637 Hägersten \\ Stockholm, Sweden
}


parallel plates is squeezed by their relative motion perpendicular to the plane. A force is produced that opposes the motion of the plates (Vermuri 2000). The conventional measures adapted to reduce squeeze-film damping in microscale resonators include (a) sealing the vibrating structure in a rarefied air (b) designing structures where slide film damping dominates (c) perforating the movable plate. Obviously, the objective to seal the vibrating structure in a rarefied air is discarded for atmospheric pressure sensors. The application of slide film damping is confined to specific structures where the motion of the movable plate is parallel to the substrate. Besides, the controllability of damping ratio is quite poor in this case. On the contrary, implementing perforations in resonators has been adopted as a standard solution to reduce squeeze-film damping. They are easy to implement in micro-scale resonators and provide an efficient etching with high controllability of damping and stiffness (Pandey 2006).

Several milestones in previously reported works have been investigated. They elucidate the use of perforations in micro-scale structures to reduce the squeeze-film damping effect, based on Reynolds' equation. Škvor (1967), in his approach, neglected the boundary condition and damping effect of air through the perforations. This limitation was overcome by Kim et al. (1999). They re-estimated the theoretical damping force by addressing the limitation in Škvor's approach. They stated that zero pressure boundary condition was one of the major setbacks in underestimating the net damping force. Besides, in their approach, they assumed that when the thickness of a movable plate is comparable to the air gap between the two parallel plates, flow through the perforations is an important consideration in the computation of net viscous damping. The Reynolds' equation was modified by Bao et al. (2003) to couple the effect of squeeze film damping through the perforations and the air gap between the two parallel plates. The assumption held by this approach was applicable for an incompressible flow in perforated structures, operating at low frequencies. These results were validated numerically using ANSYS (2D) and experimentally with that of Kim et al. (1999). Pandey et al. (2007) developed a compact analytical formula for calculating the damping and spring force in perforated structures. This approach is employed in the optimization of the resonator design in this paper. The governing equations used in Pandey et al.'s (2007) work are based on the approach followed by Bao et al. (2003) to model the perforation effects. In their approach, the boundary conditions and damping effect of air flow have been considered to realize the net damping coefficient. The resonant pressure sensor reported in this work relies on the damping effect of the surrounding fluid that relates directly to the resonator's quality factor $(Q)$. The variation in quality factor is used to sense the pressure rather than the variation in resonant frequency as the resonant frequency has a complex dependence on pressure.

The present work is dedicated to the modeling, fabrication and characterization of a resonant pressure sensor, based on the change of the quality factor with pressure. The structure is monolithically integrated with CMOS. Following the previous work of Pandey et al. (2007), we have optimized the design for a perforated square plate pressure sensor. In Section 2, the principle of operation of a resonant sensor is reviewed. The methodology employed to optimize the resonator design has been laid out in Section 3. As the sensor is targeted to enhance its sensitivity at atmospheric pressure, the analysis of squeeze film damping is carried out to formulate an analytical model of the device in MATLAB. The optimization of the formulated model is executed by comparing the sensitivity of two geometries with different air gaps between the parallel plates. This is supported by observing the trend of the quality factor with the variation in Knudsen number (defined as the ratio of the mean free path to the surrounding pressure), which in turn characterizes the surrounding pressure into two main operating regimes for our study. The numerical damping is performed by finite element simulations in COMSOL Multiphysics to calculate a net damping coefficient. A brief overview of the design and release of the test chip is presented in Section 4. This is followed by Section 5 which illustrates the experimental setup that is used to characterize the pressure sensor. The experimental conditions used to obtain the frequency response of the device are stated. In Section 6, the experimental measurements are used to derive the quality factor of the sensor as a function of surrounding pressure. This is done with the help of a behavioral model in Cadence. Finally, in Section 7, the characterization results and a comparative study of the experimental and simulation results of the prototype resonant pressure sensor are detailed which demonstrate the validity of the device at atmospheric pressure. 


\section{Device operation}

The dynamic performance of a MEMS structure can be described as a spring-mass damped vibrating system. Fig. 1 illustrates a simplified schematic of MEMS resonator that is followed by an explanation of its mechanical behavior.

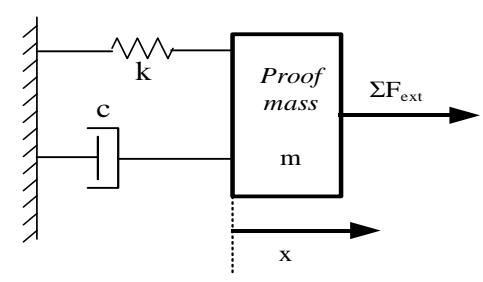

Fig. 1 Schematic of mass resonator

By applying Newton's second law of motion to this system, we achieve the following second-order differential equation (Senturia 2001) expressed as

$m \cdot \ddot{x}+c_{\text {total }} \cdot \dot{x}+k \cdot x=\Sigma F_{\text {ext }}$

Where $F_{\text {ext }}$ is the external force applied to the resonator, $m$ is the effective mass, $c_{\text {total }}$ is the net damping coefficient, and $k$ is the spring constant of the system. Here $x, \dot{x}, \ddot{x}$ are displacement, velocity ad acceleration of the system. Without application of an external force, when a single initial force is applied to the resonator, the system oscillates at its fundamental or oscillation frequency. The oscillation frequency $f_{r}$ depends on the parameters of the resonator and it is written as

$f_{r}=\frac{1}{2 \Pi} \times \sqrt{\frac{k}{m}}$

When no external force is applied to the resonator, several damping mechanisms represented by the parameter $c_{\text {total }}$ expressed in Equation (1) offsets the vibrations, annulling the vibrations completely. The quality factor of the system, $Q$ can be inferred as

$Q=\frac{1}{2 \zeta}=\frac{\sqrt{m k}}{c_{\text {total }}}=\frac{m \omega_{r}}{c_{\text {total }}}$

Where $\zeta$ is the damping ratio and $\omega_{r}$ is the angular frequency of the system at the resonant frequency.

A condition of resonance is achieved when an external harmonic force is applied to the resonator with the same frequency as the resonator's natural frequency. The amplitude of these vibrations increases, however as a result of damping, the amplitude of vibration settles at a certain level. With Laplace transform notation, Equation (1) can be converted into a second order transfer function as expressed in Equation (4).

$\frac{X(s)}{F(s)}=\frac{1}{s^{2}+s \cdot \frac{c_{\text {total }}}{m}+\frac{k}{m}}=\frac{1}{s^{2}+s \cdot \frac{2 \pi f_{r}}{Q}+\left(2 \pi f_{r}\right)^{2}}$

The next section describes the procedure for optimizing the resonator design, in order to achieve the minimum damping coefficient, and hence maximize the quality factor of the device. 


\section{Design Methodology}

This section details the methodology applied to design the targeted CMOS MEMS resonant pressure sensor. Quality factor, $Q$ of the device is primarily dependent on the viscosity. This is explained in terms of Knudsen number $K_{n}$, which in turn, is related to the ambient pressure. The explanation is detailed in Sections 3.1 and 3.2. The results obtained from the analytical model were compared to that obtained by finite element modeling to optimize the designed device.

\subsection{Calculation of Squeeze film damping from Analytical Model}

The degree of rarefaction of gases is characterized by Knudsen number $K_{n}$. This parameter is defined as the ratio of mean free path, $\lambda$ (average path traversed by an air molecule before colliding with another molecule) and the characteristic length of flow of the device $h_{0}$ (depends on the air gap thickness between the movable plate and fixed substrate). It is expressed as

$K_{n}=\lambda / h_{0}=\lambda_{0} P_{0} / P_{a} h_{0}=0.0068 /\left(P_{a} \times h_{0}\right)$

The mean free path, at any other pressure other than ambient pressure $P_{0}$, has been expressed as a ratio of its value at ambient pressure $\lambda_{0}$ in Equation (5) (Younis MI 2011). Based on the value of $K_{n}$, the fluid flow is classified into one of the four regimes (depending on the characteristic length of the device) i.e. continuum flow, slip flow, transition flow and molecular flow regimes. In the continuum flow regime where $P_{a}>272000 \mathrm{~Pa}, K_{n}<0.01$, air damping is essentially independent of the surrounding pressure. As pressure reduces, the mean free path increases and becomes comparable to the characteristic flow length i.e. the air gap between the electrodes. This results in a rise in the Knudsen number (Pandey 2008). In the slip flow regime where $272000 \mathrm{~Pa}>P_{a}>27200 \mathrm{~Pa}, 0.01<K_{n}<0.1$, phenomena such as temperature jump and velocity slip occur due to change in the boundary conditions. This requires a modification of the continuum model. At very low pressures where $27200 \mathrm{~Pa}>P_{a}>272 \mathrm{~Pa}, 0.1<K_{n}<10$, the mean free path is comparable to the characteristic length of the device (Pandey 2008). As a result, both structural and film damping effects contribute equally to the computation of the damping coefficient. In the free molecular flow regime where $P_{a}<272 \mathrm{~Pa}, K_{n}>10$ the individual gas molecules-device interactions become the dominant loss mechanism and air stops behaving like a viscous fluid (Kaajakari 2009).

A summary of the equations developed by Pandey et al. (2007) and Veijola (2006) are simplified for the proposed square plate membrane and stated underneath to justify the foundation of the suggested analytical model. The fundamental principle that has been used in his work is to employ modified Reynolds' equation to calculate the net damping coefficient. The vibrating movable plate is assumed as a set of uniformly distributed cells. The flow around the internal perforations near the boundary of the structure exhibits an asymmetric flow pattern due to the squeezefilm flow. This occurs due to the pressure gradient that exists along the length of the oscillating plate. This assumption holds rational when pressure gradient drops as the perforation ratio, defined as the ratio of perforation size to perforation pitch, is large (Pandey et al. 2007). The governing equations incorporate the loss through perforations and the varying losses at the boundary as well as in the interior of the sensor. Fig. 2 shows a schematic model of the proposed sensor structure to represent the squeeze-film damping effect. Fig. 2(a) illustrates the proposed design of a square plate membrane of length $L$ along the x-axis, width $W$ along the y-axis, thickness $T_{p}$ along the z-axis and air gap $h_{0}$ between the movable plate and fixed substrate. Since $W=L$ for the proposed structure, the plate aspect ratio, $W / L=1$ for the equations stated underneath. The plate contains $N_{h}=M \times N$ uniformly distributed square perforations; each perforation sized $L_{h}$ and pitch $q$ along both $\mathrm{x}$ and y coordinates respectively. The dynamics of air flow through a typical cell has been shown in Fig. 2(b). As shown, by comparing the resistance of circular pipe and square channel of the same length, we get an equivalent perforation radius, $b=1.096 L_{h} / 2$ and cell radius $a=q / \sqrt{ } \pi$ (Veijola 2006). 


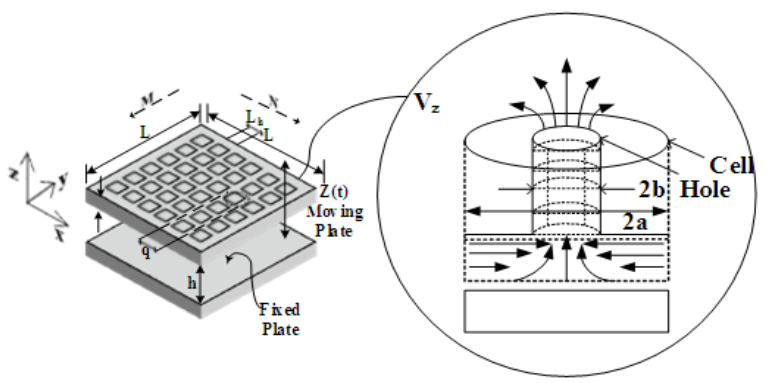

(a)

(b)

Fig. 2 Schematic diagram for squeeze-film damping in the perforated system under consideration:

(a) Oscillating perforated plate and the fixed plate in isometric view

(b) Airflow dynamics under a typical perforation-cell combination (Adapted from [Pandey 2007])

The corresponding damping coefficient due to squeeze-film in the viscous regime (Pandey 2007) of pressure is expressed as

$c_{\text {squeeze }}=\frac{16 \sigma}{\pi^{6}} \frac{P_{a} L^{2}}{\omega_{r} h_{0}} \sum_{m, n=o d d} \frac{\left.\left[\left(\Gamma^{2} / \pi^{2}\right)+m^{2}+n^{2}\right)\right]\left(4-f_{\text {perf }}\right)}{(m n)^{2}\left\{\left[\Gamma^{2} / \pi^{2}+m^{2}+n^{2}\right]^{2}+\sigma^{2} / \pi^{4}\right\}}$

Where $m, n=\{1,3,5 \ldots\}$ are the harmonic modes of the resonator (Darling 1998), $\sigma=\frac{12 \mu \omega L^{2}}{h_{0}^{2} P_{a} Q_{c h}}$ is the squeeze number that captures the compressibility effect, $P_{a}$ is the ambient pressure and $h_{0}$ is the air gap between the movable plate and fixed plate. $\Gamma=L^{2} \sqrt{\frac{12 \mu}{Q_{c h} h_{0}^{3} R_{P} N_{R}}}$ is a constant that captures perforation effect, $f_{\text {perf }}$ is the force through each perforation, $\mu$ is the viscosity of air at ambient conditions, and $Q_{c h}$ is the flow rate factor that produces rarefaction effect in the flow through parallel plates and substrate, $R p$ is the net resistance in a single perforation cell, and $N_{R}$ is the contribution of perforations in the interior and boundaries of the membrane. The term $P_{a}$ appearing the numerator of Equation (6) simplifies away with that included as a function of $\sigma$ in the denominator. However, this term appears in the last term of the denominator inside the summation series, which establishes a relation between $c_{\text {squeeze }}$ and $P_{a}$.

The damping coefficient due to the loss through perforations (Veijola 2006), $c_{\text {perforation }}$ is expressed as

$c_{\text {perforation }}=8 \pi \mu\left(\frac{T_{p}}{Q_{t h}}+\Delta_{E} b\right) \times N_{h}$

Where $Q_{t h}$ is the relative flow rate to account for the rarefaction effect in the perforation, $\Delta_{E}$ is the relative elongation of the perforation length due to open end effects and $N_{h}$ is the total number of perforations in the movable plate. Finally, net damping constant is expressed as

$c_{\text {total }}=c_{\text {squeeze }}+c_{\text {perforation }}$

The squeeze area reduces for higher perforation ratios and eventually the loss through the perforations is a dominant factor in fluid damping.

For viscous damping during oscillations, assuming the displacement of the resonator is $x=A \sin \omega t$, where $A$ is the amplitude of oscillation, $\omega$ is the angular frequency of oscillation of the membrane and $t$ is the time, the damping force is $F_{d}=c_{\text {total }} \dot{x}$; where $\dot{x}$ is the velocity expressed as $\dot{x}=A \omega \cos \omega t$. If $m$ is the effective mass of the vibrating system, then the computed quality factor (recalled from Equation (3)) can also be expressed as 
$Q=2 \pi \frac{E_{\text {input }}}{\Delta E_{\text {loss }} \text { per cycle }}=2 \pi \frac{\frac{1}{2} m A^{2} \omega_{r}^{2}}{\int_{0}^{2 \pi / \omega_{r}} F_{d} \dot{x} d t}=\frac{m \omega_{r}}{c_{\text {total }}}$

Where $F_{d}$ is the damping force due to viscous damping (Pandey 2007). The equations mentioned above have been employed to formulate a user-defined MATLAB script to compute the total squeeze film damping coefficient and hence, obtain the quality factor for the structure.

\subsection{Sensor optimization using analytical model}

From the above discussion, we have inferred that the quality factor of a MEMS pressure sensor primarily depends on the viscosity of the surrounding fluid i.e. air; which is dependent on pressure. The damping coefficient of the fluid and that due to loss through perforations depend on several factors such as thickness of the movable plate $\left(T_{p}\right)$, the air gap between the movable plate and fixed plate $\left(h_{0}\right)$ and number of perforations in the movable plate $\left(N_{h}\right)$ (Banerji 2015).

A script has been used to generate a mesh for the design mentioned above, and the corresponding function has been adapted to call several variables as per the user's requirements. The output performance parameters such as quality factor, net damping coefficient and capacitance of the device were optimized against a wide spectrum of input parameters i.e. length of each perforation, the thickness of the perforated movable plate and ambient pressure to minimize the energy loss of the system. For instance, the length of each perforation has been set to $18 \mu \mathrm{m}$ after varying the size of each perforation (from $2 \mu \mathrm{m}$ to $50 \mu \mathrm{m}$ ) with the capacitance, net damping coefficient and quality factor of the device. Simulation results show that any deviation from $18 \mu \mathrm{m}$ leads to a reduction in the effective plate area. There is a decrease in the capacitance and effective mass of the device followed by a reduction in its quality factor. However, tradeoffs such as an increase in actuation voltage are detrimental effects of increasing the air gap and perforations for a given cross-sectional area. Although a greater perforation area can enhance the quality factor, the total surface area of the plate suffers an overall reduction that results in a reduction of the device capacitance. However, the decrease in the device capacitance is limited by the difficulty for the electronics to perform the measurements.

The dynamics of two representative geometries; Geometry I: $140 \mu \mathrm{m} \times 140 \mu \mathrm{m}$ with an air gap of $2.5 \mu \mathrm{m}$ and Geometry II: $250 \mu \mathrm{m}$ x $250 \mu \mathrm{m}$ with an air gap of $4 \mu \mathrm{m}$ are evaluated to optimize the performance parameters against the structural parameters of the plates. For convenience, the two geometries, taken into account, are addressed as Geometry I and Geometry II, throughout the text. We have simulated several geometries to select the mentioned structures that cover the reasonable range of devices. The maximum dimensions of the membrane are limited by reliability issues while the minimum dimensions are restricted by low capacitance and parasitic degradation. The performance parameters of the respective structures have been compared at atmospheric pressure to evaluate the viscous damping. This comparative analysis was performed to select the structure that exhibits the maximum sensitivity in the viscous regime of air pressure. The comparison of the parameters of the MEMS structures, taken into consideration, is tabulated in Table 1.

Table 1 Comparison of parameterization and dynamics of two geometries at standard atmospheric pressure

\begin{tabular}{|c|c|c|c|c|}
\hline \multirow[b]{2}{*}{ Symbol } & \multirow[b]{2}{*}{ Description } & \multicolumn{2}{|c|}{ Values } & \multirow[b]{2}{*}{ Units } \\
\hline & & Geometry I & Geometry II & \\
\hline \multicolumn{5}{|c|}{ Independent variables } \\
\hline$L$ & Length of the square plate & $140 \times 10^{-6}$ & $250 \times 10^{-6}$ & $\mathrm{~m}$ \\
\hline $\boldsymbol{W}$ & Width of the square plate & $140 \times 10^{-6}$ & $250 \times 10^{-6}$ & $\mathrm{~m}$ \\
\hline$T_{p}$ & Thickness of the square plate & \multicolumn{2}{|c|}{$8 \times 10^{-6}$} & $\mathrm{~m}$ \\
\hline$L_{h}$ & Length of perforation & \multicolumn{2}{|c|}{$18 \times 10^{-6}$} & $\mathrm{~m}$ \\
\hline
\end{tabular}




\begin{tabular}{|c|c|c|c|c|}
\hline \multirow[b]{2}{*}{ Symbol } & \multirow[b]{2}{*}{ Description } & \multicolumn{2}{|c|}{ Values } & \multirow[b]{2}{*}{ Units } \\
\hline & & Geometry I & Geometry II & \\
\hline$N_{h}(N \times M)$ & Number of perforations & $36(6 \times 6)$ & $121(11 \times 11)$ & - \\
\hline $\boldsymbol{h}_{0}$ & Air gap thickness & $2.5 \times 10^{-6}$ & $4 \times 10^{-6}$ & $\mathrm{~m}$ \\
\hline$\rho_{\text {plate }}(A l-W-A l)$ & Effective density of plate & \multicolumn{2}{|c|}{6500} & $\mathrm{~kg} / \mathrm{m}^{3}$ \\
\hline$s$ & Spacing between two perforations & \multicolumn{2}{|c|}{$4 \times 10^{-6}$} & $\mathrm{~m}$ \\
\hline $\boldsymbol{\mu}$ & Dynamic viscosity coefficient of air & \multicolumn{2}{|c|}{$1.67 \times 10^{-5}$} & Pa-s \\
\hline$\omega_{r}$ & Angular frequency of oscillation & \multicolumn{2}{|c|}{$103.6 \times 10^{3}$} & $\mathrm{~Hz}$ \\
\hline$\varepsilon_{0}$ & Vacuum permittivity & \multicolumn{2}{|c|}{$8.854 \times 10^{-12}$} & $\mathrm{~F} / \mathrm{m}^{2}$ \\
\hline$\varepsilon r$ & Relative permittivity of air & \multicolumn{2}{|c|}{1} & - \\
\hline $\boldsymbol{A}_{e}$ & Area of the plate & $7.936 \times 10^{-9}$ & $23.3 \times 10^{-9}$ & $\mathrm{~m}^{2}$ \\
\hline$\lambda$ & Mean free path of gas molecules & \multicolumn{2}{|c|}{$67.11 \times 10^{-9}$} & $\mathrm{~m}$ \\
\hline \multicolumn{5}{|c|}{ Dependent Variables } \\
\hline$m$ & Effective mass & $4.127 \times 10^{-10}$ & $1.21 \times 10^{-9}$ & $\mathrm{~kg}$ \\
\hline $\boldsymbol{k}$ & Spring stiffness constant & 174.86 & 513.29 & $\mathrm{~N} / \mathrm{m}$ \\
\hline$Q$ & Quality factor & 63 & 96.5 & - \\
\hline$K_{n}$ & Knudsen number & 0.0268 & 0.0168 & - \\
\hline Cap & Capacitance & $28.11 \times 10^{-15}$ & $51.57 \times 10^{-15}$ & Farad \\
\hline$S$ & Sensitivity & -0.0413 & -0.0164 & - \\
\hline
\end{tabular}

The input parameter i.e. Pressure $\left(P_{a}\right)$ has been varied to observe the variation in the quality factor of the device and hence evaluate the overall sensitivity.

\subsubsection{Variation of the Quality factor $(Q)$ with Pressure $\left(P_{a}\right)$}

The variation of quality factor for the two geometries taken into consideration has been studied by varying the Knudsen number and the surrounding pressure. The air flow regimes have been classified according to the characteristic length of Geometry I. The trend observed in Fig. 3 suggests that in the slip flow regime, for both the geometries, the variation in the quality factor is extremely low at lower Knudsen numbers. However, we can observe that Geometry I shows a higher variation of $Q$ with $K_{n}$ i.e. greater sensitivity than that of Geometry II. 


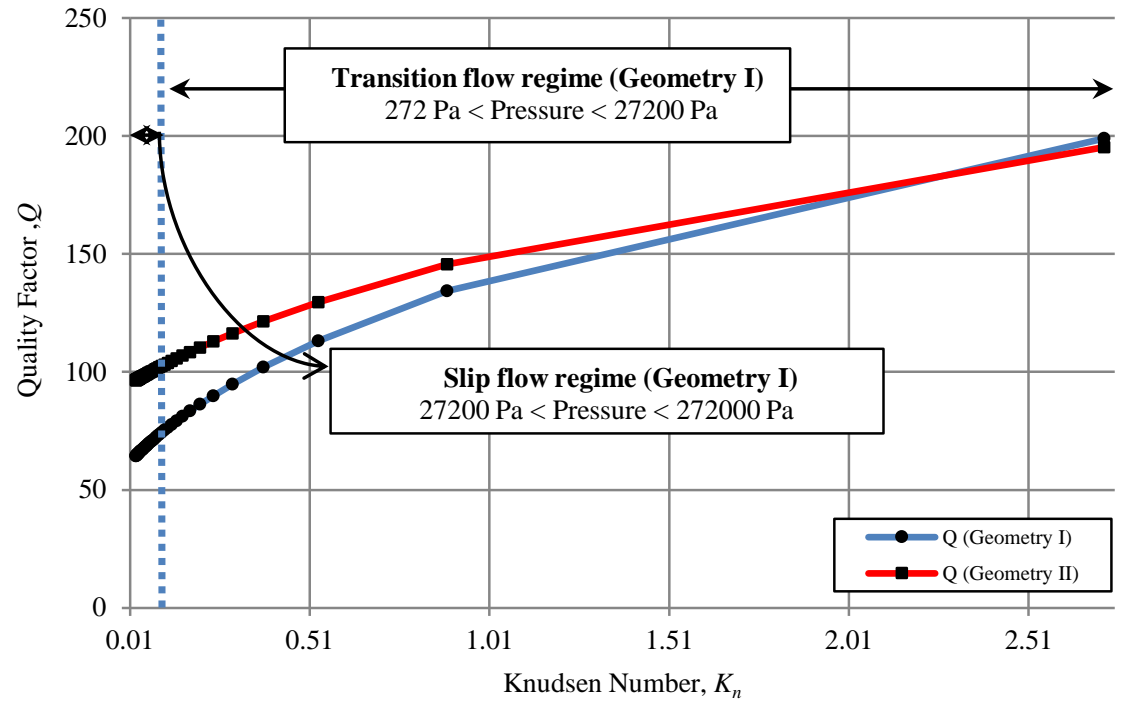

Fig. 3 Variation of the quality factor $(Q)$ with Knudsen number $\left(K_{n}\right)$ for the two geometries

By varying the quality factor with pressure, keeping the characteristic length of the device constant, in Fig. 4, we can distinguish the two principal operating regions of pressure (taking the characteristic length of Geometry I into account). In the slip flow and transition flow regime, the quality factor is proportional to an inverse of the square root of the surrounding pressure and $K_{n}$ is estimated to be varying between 0.01 and 10 . At lower surrounding pressures, the variation in the quality factor of the structures shoots considerably high. This characteristic trend is employed for the extraction of the quality factor from experimental results and discussed later in Section 6.

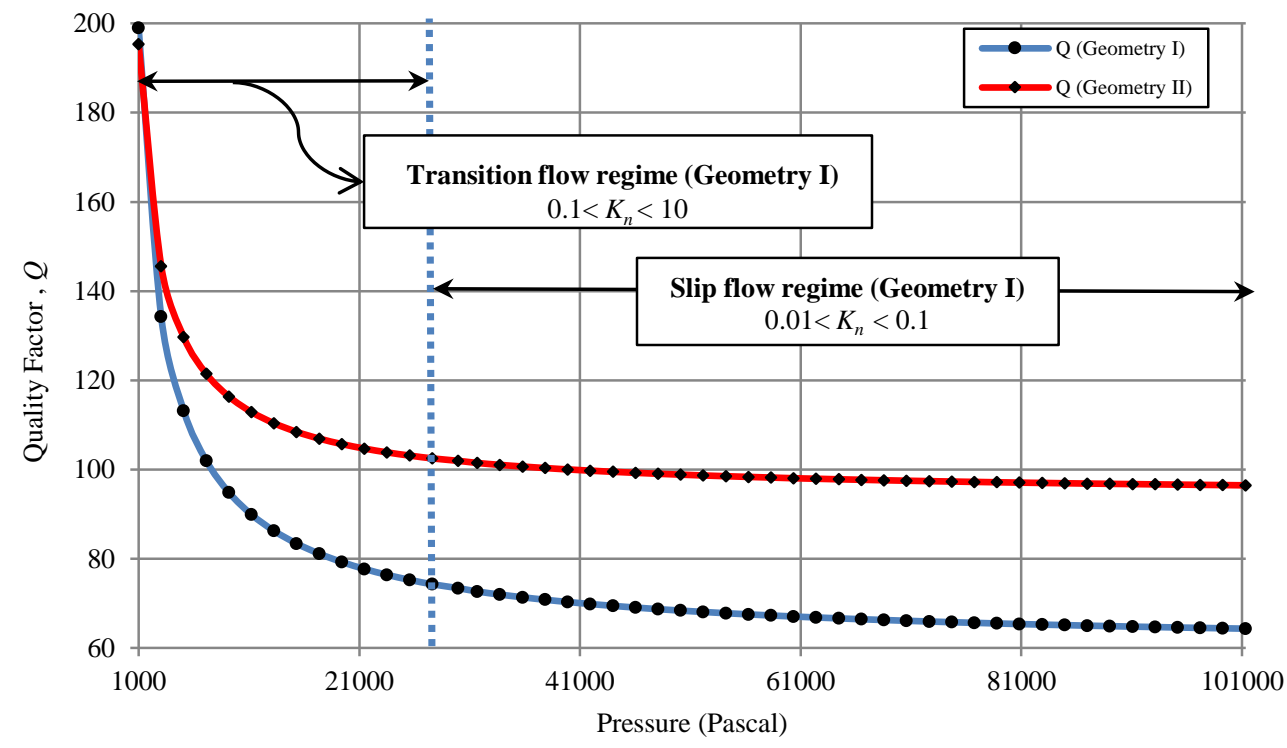

Fig. 4 Variation of the quality factor $(Q)$ with pressure $\left(P_{a}\right)$ for the two geometries

The quality factor was observed over a broad range of perforation length i.e. from $2 \mu \mathrm{m}$ to $18 \mu \mathrm{m}$ for Geometry I and from $2 \mu \mathrm{m}$ to $50 \mu \mathrm{m}$ for Geometry II. The range of perforation length has been varied according to the available 
surface area in the two geometries. The variation of $Q$ with $L_{h}$ is illustrated in Fig. 5. The spacing between two perforations is calculated as a function of the number of perforations in the membrane. In Geometry II, the quality factor, in general, increases linearly with the length of perforation until a saturation point after which the curve follows

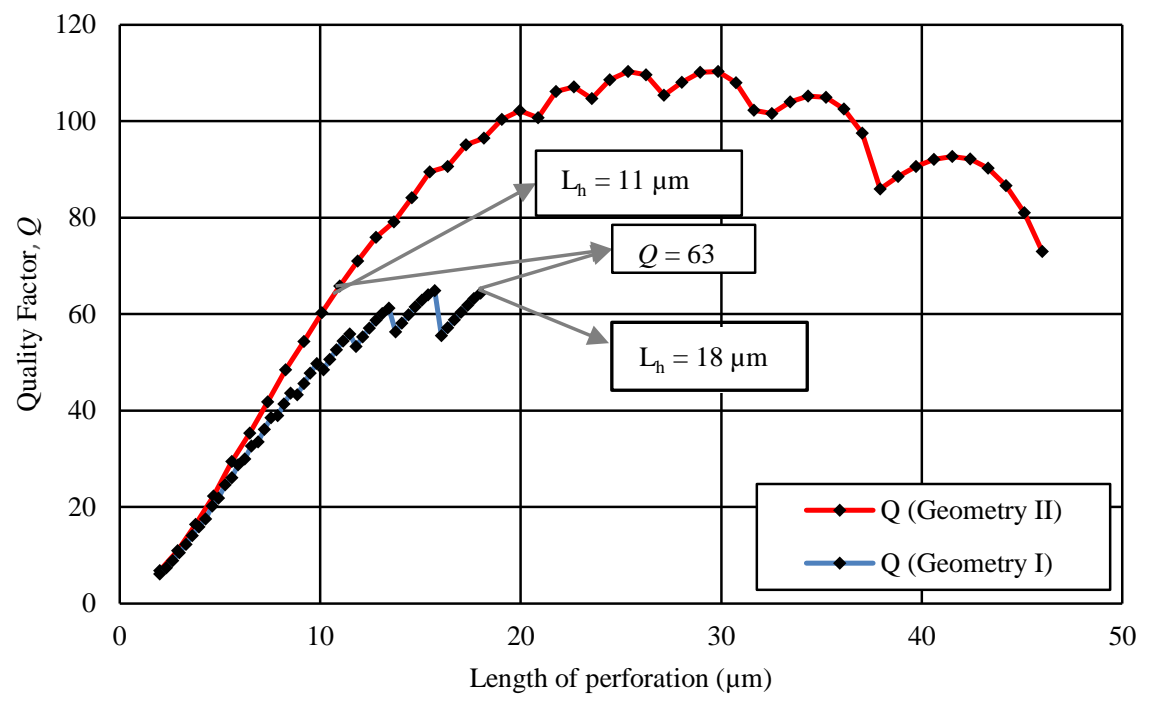

Fig. 5 Variation of quality factor $(Q)$ vs. length of perforation $\left(L_{h}\right)$ for the two geometries

a declining trend. The non-monotonic behavior and reduction of $Q$ after the saturation point occurs due to the elimination of perforations with an increase in $L_{h}$ that varies as a function of the spacing between the perforations. Any deviation beyond $18 \mu \mathrm{m}$ leads to a reduction in the effective plate area, which in turn decreases the plate capacitance. From Fig. 5, we can deduce that with the same quality factor i.e. equivalent energy dissipation for both the geometries, the length of perforation is $18 \mu \mathrm{m}$ and $11 \mu \mathrm{m}$ for Geometry I and II respectively (Banerji 2015). 


\subsubsection{Computation of Sensitivity}

The sensitivity of the sensor has been computed quantitatively as $S=(\Delta Q / Q) /(\Delta P / P)$. From Fig. 3 and Fig. 4 , we can deduce that the sensitivity is approximately -0.04 for Geometry I and approximately -0.016 for Geometry II at atmospheric pressure whereas approximately -43 for Geometry I and approximately -22.9 for Geometry II at low

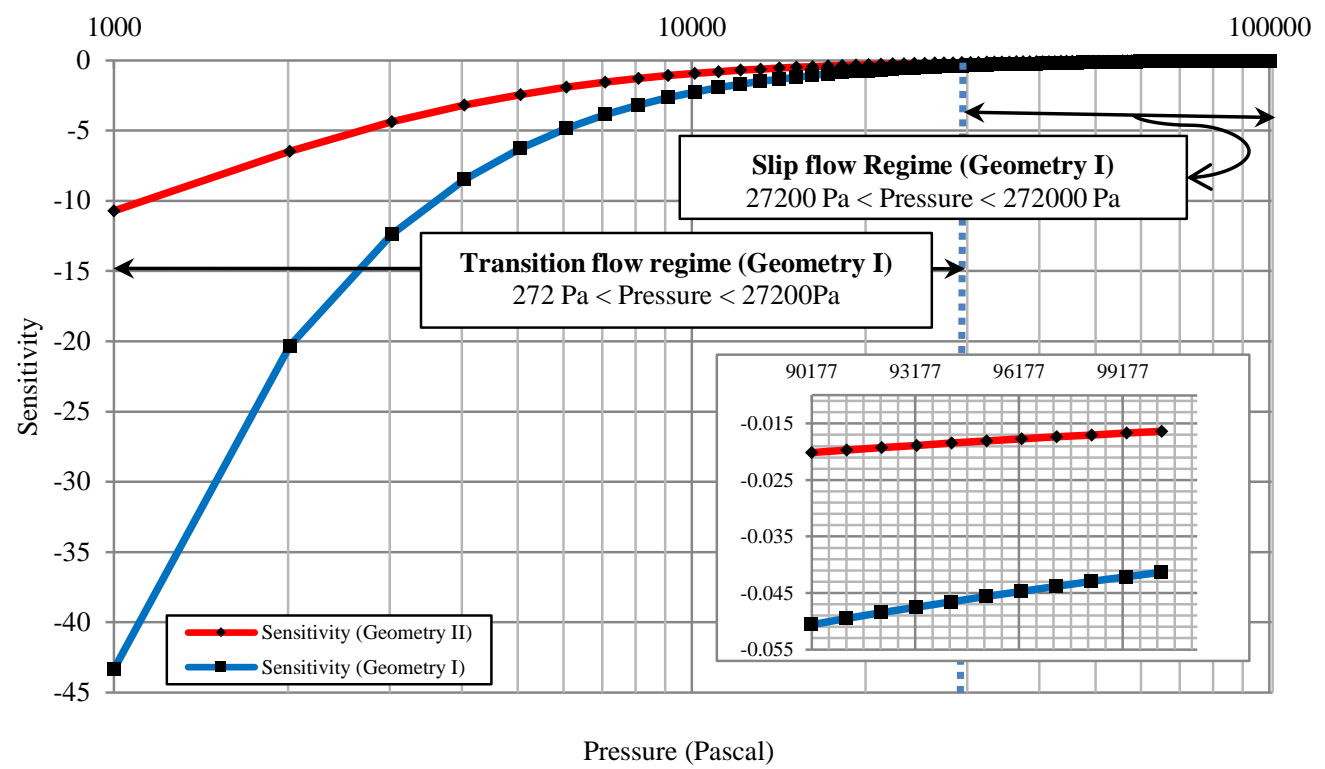

Fig. 6 Sensitivity of sensor for the two geometries

pressures of $1 \mathrm{kPa}$. The inset in Fig. 6 shows a zoom of the sensitivity of the structures at atmospheric pressure range to justify that the Geometry I yields a higher sensitivity than Geometry II.

Fig. 3 shows that at atmospheric pressure, Knudsen number of Geometry I is 0.026 which is greater than that of Geometry I having $K_{n}$ of 0.016 (As this point is beyond the atmospheric pressure for Geometry I, it is not shown in the illustration). Thus, the device behavior of Geometry I enters the slip flow regime faster than that of Geometry II. As the variation of quality factor is independent of the surrounding pressure at continuum flow regime, the variation of quality factor is higher for Geometry I than Geometry II, at a constant interval of $1 \mathrm{kPa}$. This implies that Geometry I has a higher sensitivity than Geometry II.

From the analytically performed optimization procedure, we can conclude that, to enhance the device performance i.e. the sensitivity, either the mean free path of air (by reducing surrounding pressure) or the air gap thickness needs to be reduced. Since the targeted study intends to enhance the sensitivity of the device at atmospheric pressure while keeping the device capacitance and quality factor at optimum values. The performance parameters of several structures have been varied against their structural parameters to optimize the device. Finally, Geometry I with lateral dimensions of $140 \mu \mathrm{m} \times 140 \mu \mathrm{m}$, thickness $\mu \mathrm{m}$ of $8 \mu \mathrm{m}$ and an air gap thickness of $2.5 \mu \mathrm{m}$ is proposed as the optimized structure.

\subsection{Computation of Quality factor $(Q)$ for optimized device using Finite element modeling (FEM)}

The dynamic performance of the device is observed for a surrounding pressure, ranging from $100 \mathrm{~Pa}$ to atmospheric pressure. Unlike the continuum flow regime where $K_{n}<0.01$, the device behavior deviates from the standard Reynold's equation for $0.01<K_{n}<0.1$ in slip flow regime as discussed earlier in Section 3.2.1. In the transition flow regime, where $0.1<K_{n}<10$, the mean free path of the molecules becomes comparable to the air gap between the electrodes. The analysis becomes more complicated as the intermolecular collisions are comparable to the collisions of the gas molecules, and the continuum model is no longer valid (Pandey 2008). As squeeze film damping is negligible below $272 \mathrm{~Pa}$, the structural damping is the only damping mechanism accounted for, at such low pressures 
in the molecular flow regime. The analytical model fails as it underestimates the damping at such low pressures. Finite element modeling in COMSOL Multiphysics is used to overcome this limitation.

\subsubsection{FEM analysis of damping mechanisms}

The suggested analytical model holds valid in the slip flow and transition flow regime. The structural damping effect is prevalent in the molecular flow regime i.e. $P_{a}<272 \mathrm{~Pa}$ while both structural and squeeze film damping effects are implemented for emulating the device behavior in the transition flow regime i.e. $272 \mathrm{~Pa}<P_{a}<27200 \mathrm{~Pa}$. The structural and film damping effects are employed to estimate the total damping coefficient and compute the net $Q$ of the device. The theory and analysis for calculation of the squeeze film damping effect and perforation losses have been discussed in Sections 3.1 and 3.2. A brief overview of the structural losses is given underneath.

\section{- Anchor losses}

A non-negligible part of the mechanical energy stored in the device leaks into the anchors and the substrate that serve as a mechanical support for the vibrating membrane (Kaajakari 2009). Anchor losses form a dominant structural damping mechanism in microresonators. They depend on the mode of anchoring and the mode of operation of the vibrating membrane. The objective to reduce the energy dissipation from anchors is to anchor the resonator at places of minimum displacement (Rawat 2013).

We have employed a flexural-mode resonator for the optimized structure. In this case, the square vibrates out-of-plane orthogonal movements (Basu 2011). The suspension beams are attached to the anchors that form the nodal points of resonance (Khine 2010). Quantitative computations of damping for such structures are extremely challenging which justify the non-convergence of the FEM simulations with the developed analytical model at pressures below $272 \mathrm{~Pa}$. The anchors are attached to the corners of the optimized structure. Studies have shown that a lower leakage of acoustic power occurs in this arrangement compared to the arrangement where the anchors are attached to the center of the vibrating membrane (Rawat 2013). Fig. 7 illustrates the layout of the pressure sensor drawn in Cadence design tools that were further imported to COMSOL Multiphysics to evaluate the quality factor of the device.

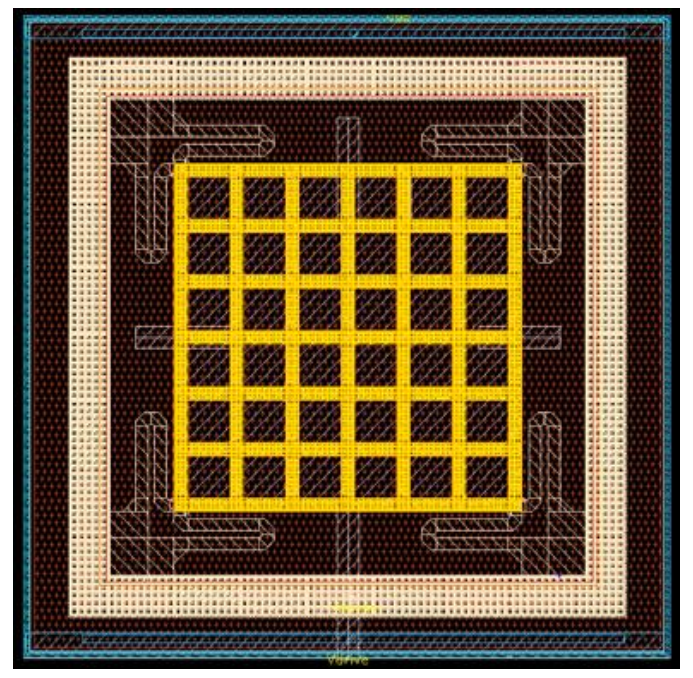

Fig. 7 Layout of optimized resonant pressure sensor

\section{- Intrinsic losses}

Selection of the right material for the designed resonant sensor is critical for minimizing the structural losses. Intrinsic losses are the least dominant energy losses present in a micro-resonator (Brotz 2004). They originate from internal friction due to the heat generation at the atomic level. As these losses are dependent on a large number of physical 
mechanisms such as vibration frequency, vibration mode, and temperature, the quantitative analysis is again very complicated.

The elastic damping losses for a material is expressed in Equation (10).

$T=E S+\mu_{\Upsilon} \frac{\partial S}{\partial t}=E S+j \omega \mu_{\Upsilon} S$

where $T, E$, and $S$ are the stress, Young's modulus for the material and strain respectively (Kaajakari 2009). The additional velocity-dependent viscous damping term, $\mu_{\Upsilon} \partial S / \partial t$ is introduced to correlate the viscous term for the force acting on the resonator where $F=k x+c_{\text {total }}(\partial x / \partial t)$. Here, $\mu_{r}$ is the viscosity of the material, $\omega$ is the angular vibration frequency, and $k$ is the spring constant.

The intrinsic quality factor $Q_{i}$ due to material losses (Kaajakari 2009) is expressed as

$Q_{i}=E /\left(\mu_{\Upsilon} \omega\right)$

From the equations mentioned above, we can deduce that intrinsic losses increase with vibration frequency. Thus, a material with low Young's modulus, low density, and low viscosity should be chosen to reduce material losses.

With a low Young's modulus of $74 \mathrm{GPa}$, a density of $2700 \mathrm{~kg} / \mathrm{m}^{3}$ and viscosity of $1 \mathrm{~N}-\mathrm{s} / \mathrm{m}^{2}$, Al has been chosen as the material for the optimized device. Tungsten, the material for via, is used to increase the overall quality factor by increasing the effective mass.

The designed structure has been simulated in COMSOL Multiphysics to evaluate the structural and viscous damping losses. Fig. 8 shown below illustrates the effect of net force on the designed square plate sensor where the movable structure is displaced from its original position with respect to the substrate that will be further evaluated to estimate the quality factor in next section.

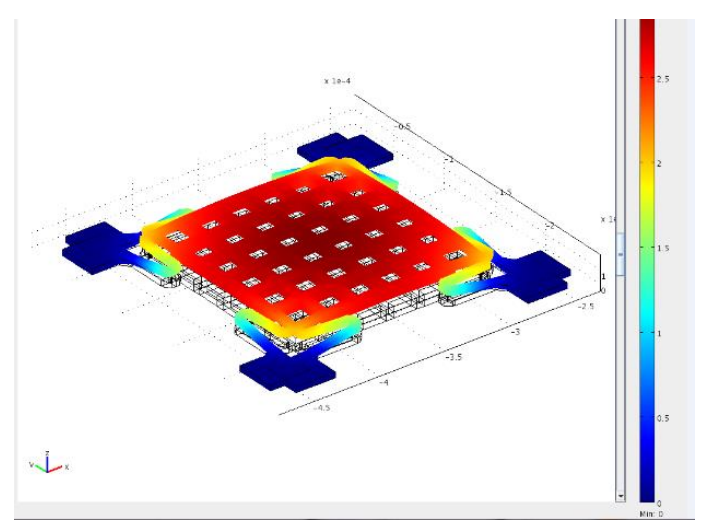

Fig. 8 Effect of damping force on designed resonant pressure sensor in COMSOL Multiphysics

\subsubsection{Estimation of Quality factor $(Q)$ of pressure sensor}

We have made use of the module in COMSOL Multiphysics, which includes structural and film damping. This module, by default, solves for the structural and fluid damping between the movable plate and the substrate (COMSOL Multiphysics MEMS Module 2008).

The membrane for designed pressure sensor imported from Cadence is simulated for solving the oscillation modes of the structure as an initial step. Both structural damping and film damping effects are employed to estimate an accurate quality factor of the system unlike in the case of simulations employed by analytical modeling via MATLAB, which quantifies film damping alone. The conditions for application of actuation force on the lower side of the movable plate 
and boundary conditions for film damping have been applied on both sides of the structure to evaluate the Eigen or resonant frequencies of the structure at atmospheric pressure ranging from $97.6 \mathrm{kHz}$ to $118.5 \mathrm{kHz}$. The fundamental mode of $97.6 \mathrm{kHz}$ obtained in COMSOL corresponds to that of the manufactured device i.e. $103.6 \mathrm{kHz}$. This Eigenfrequency analysis is carried out to estimate the Eigenvalue during the calculation of quality factor of the structure.

The film thickness, ambient pressure, mean free path and dynamic viscosity are set to their absolute values. Recalling Equation (8), the measure of the ratio of the stored energy and energy lost per cycle in a free oscillation is used to estimate the quality factor using the equivalent definition (COMSOL Multiphysics MEMS Module 2008).

$Q=\frac{\omega_{r}}{2 \times \delta}=\left|\frac{\operatorname{imag}(\lambda)}{2 \times \operatorname{real}(\lambda)}\right|$

Where $\omega_{r}$ is the natural angular frequency, $\delta$ is the damping factor and $\Delta \omega$ is half power width of the spectrum and $\lambda$ is the Eigenvalue. The standard Eigenvalue analysis is initiated for this purpose. Once the Eigenfrequency is known, squeeze film damping is added, and a frequency response analysis is carried out for calculating Eigenmode frequencies in an immediate vicinity of resonance. The resonant frequency is found to be close to $97.6 \mathrm{kHz}$ according to the Eigenfrequency analysis. At atmospheric pressure, the quality factor estimated from the analytical model around 63 at atmospheric pressure that obtained by finite element modeling is 55. The values of $Q$ in the slip flow regime and at higher pressures in transition flow regime calculated by the analytical model align well with that obtained from finite element modeling. However with $P_{a}<1000 \mathrm{~Pa}$, the structural damping dominates.

At extremely low pressures of $100 \mathrm{~Pa}, Q$ estimated by the analytical model is around 600 while that obtained by finite element modeling is 475 . The reduction in the quality factor of the structure by $26.3 \%$ owes to the structural damping that was neglected in the computation of net damping coefficient by the analytical model. The quality factor for the structure was computed at several pressures from molecular flow regime to slip flow regime. The data is tabulated and compared to the analytical and experimental results in Section 7.

\section{Release of test chip in CMOS MEMS micromachining process}

The designed square plate pressure sensor was manufactured in 250-nm CMOS technology after verifying the optimization procedure by finite element modeling. Thus, the design can, in general, be migrated to other technologies with suitable optimization strategies specific to the target process. In our case, it was manufactured using CMOS technology, and the release was performed at the dice level in the UPC-DEE clean room with a hydrogen fluoride (HF) based etchant to release the back-end of line (BEOL) metal layer and etch out the oxide (Fernandez 2010). The release process was performed by etching the samples using a buffered oxide etchant followed by resist removal and a rinse in methanol to reduce stiction issues. This was followed by drying the samples in an oven (Michalik 2015). The release process employed has shown to achieve a greater mass, high capacitance and stress optimization at a high level. The release of test chips was performed at different intervals to test the varying resonance frequency obtained for the frequency response of the test chips. A typical layer diagram has been illustrated in the schematic shown in Fig. 9. 


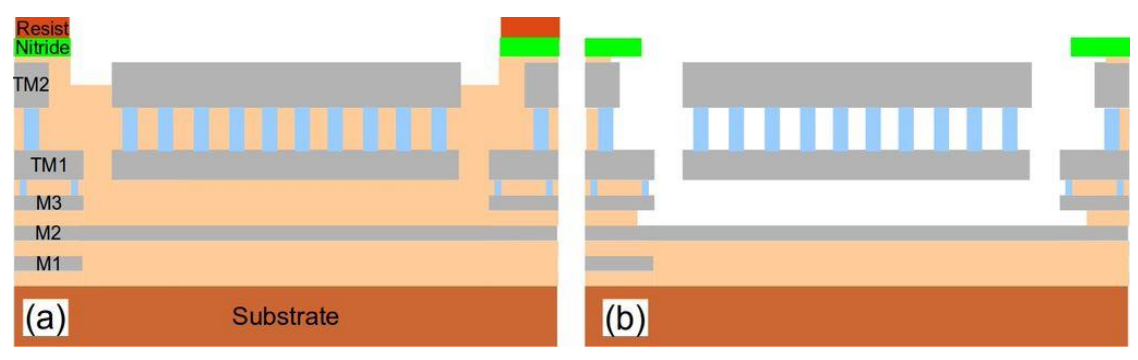

Fig. 9 Stack diagram of simplified IHP SG25H1/H3 BEOL stack diagram after wet isotropic etching till substrate (a) Pre-Release cross section (b) Post-Release cross section

Fig. 10 shows the view of the test chip released by the aforementioned process.

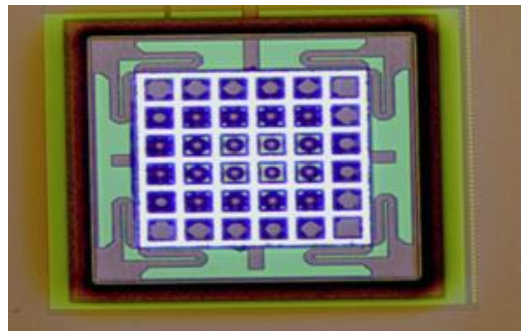

(a)

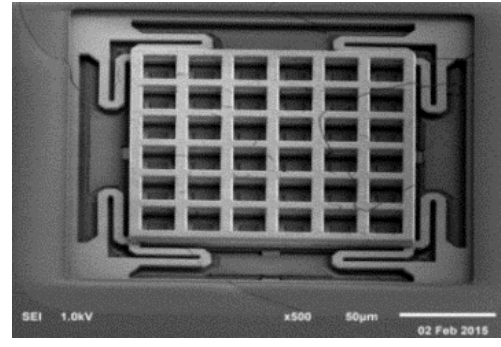

(b)

Fig. 10 View of the test Chip

(a) Microphotograph

(b) Under scanning electron microscope

\section{Experimental set-up}

As the next step, frequency response analyses were carried out for test chips released for several etching times. The released test chip illustrated in Fig. 10 was measured with an impedance analyzer available in our in-house laboratory. The experimental set-up used to obtain the measurements from the test chip is shown in Fig. 11. The measurements were taken with an Agilent 4294A precision impedance analyzer and a vacuum chamber equipped with probes and a microscope. The measurements above $2000 \mathrm{~Pa}$ were taken with $120 \mathrm{mV}$ oscillation amplitude while that below 1000 $\mathrm{Pa}$ were taken with $60 \mathrm{mV}$ oscillation amplitude to mitigate the effects of nonlinearity due to excessive amplitude vibration. The DC bias was set at $5 \mathrm{~V}$, and the frequency was varied from $100 \mathrm{kHz}$ to $110 \mathrm{kHz}$. The frequency response thus generated on the screen of the impedance analyzer was transferred to the system and an excel file was generated by the data interpolation.

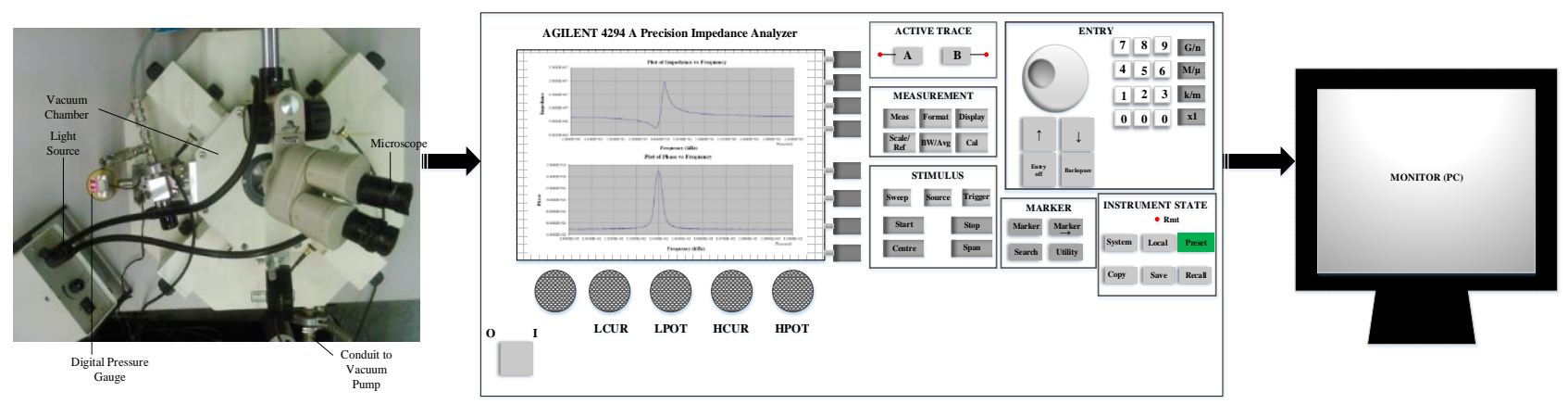

Fig. 11 Experimental set-up for obtaining frequency response from designed sensor

The quality factor of the sensor was extracted from the comparison of the frequency response (magnitude and phase) obtained from the impedance analyzer at variable ambient pressures, in the following section. 


\section{Extraction of Quality factor $(Q)$ from experimental set-up results}

The frequency response of the test chip was obtained by varying the pressure from $100 \mathrm{~Pa}$ to atmospheric pressure. The quality factors for the frequency response obtained from experimental measurements were extracted using a second order electrostatic behavioral model in Verilog-A. This behavioral model relates the total force of actuation applied on the movable membrane to the external force, acceleration, temperature and Brownian noise, thus taking non-linearity of the device into account. Following this, the extracted quality factors of the structure are plotted against varying pressures to obtain a curve fitting in MATLAB. The quality factor, $Q$ is defined as a function of pressure, $P_{a}$ in the behavioral model such that with the pressure as an input variable, $Q$ can be obtained directly. This curve fitting, as shown in Fig. 12 is performed with the experimental measurements from $100 \mathrm{~Pa}$ to atmospheric pressure.

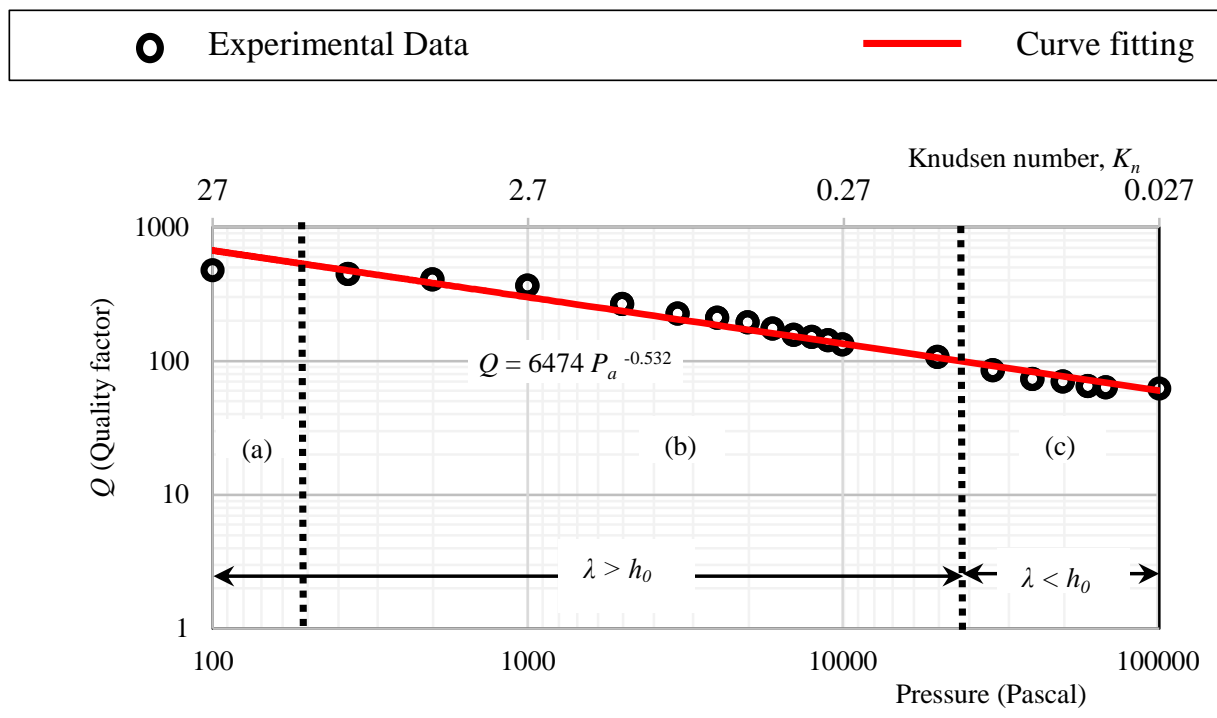

Fig. 12 Fitting of power law over (a) Molecular flow regime (b) Transition flow regime (c) Slip flow regime

As shown in Fig. 12, $Q$ of the manufactured device follows a power law with the surrounding pressure. The mean free path over the molecular and transition flow regime is greater than the characteristic length of the device while it is negligible compared to the characteristic length of the device at higher orders of pressure in the slip flow regime.

The variation of $Q$ with pressure $P_{a}$ (incorporated in the behavioral model) is expressed as

$Q=a\left(P_{a}\right)^{b}$

Where $a$ and $b$ are coefficients relating $Q$ to $P_{a}$. The frequency response is generated at variable input pressures and aligned with that obtained from experimental measurements. This is detailed in the following Section 7.

\section{Results: Comparison and validation of manufactured sensor}

The frequency response of the manufactured resonator was plotted from $100 \mathrm{~Pa}$ to atmospheric pressure at a fixed bias voltage of $5 \mathrm{~V}$. An oscillation level of $60 \mathrm{mV}$ was used for pressures under $1000 \mathrm{~Pa}$ and while $120 \mathrm{mV}$ for pressures above $2000 \mathrm{~Pa}$. The obtained measurements were aligned and simulated in Cadence using a Verilog-A model. The proposed behavior model incorporates a power equation relating $Q$ to $P_{a}$ with input coefficients generated by curve fitting toolbox in MATLAB. Fig. 13 shows the alignment of the frequency response from the experimental measurements and simulations performed in Cadence. 

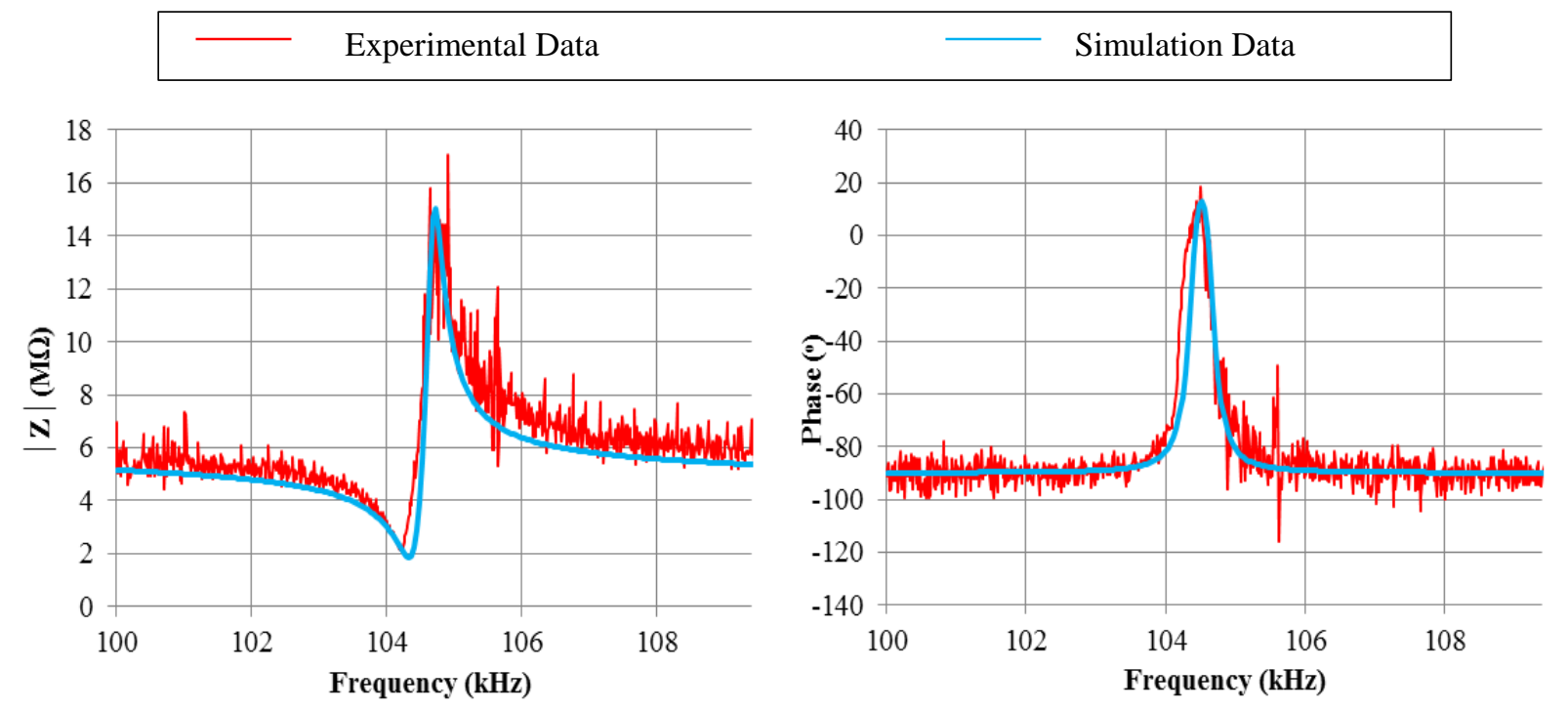

(a)
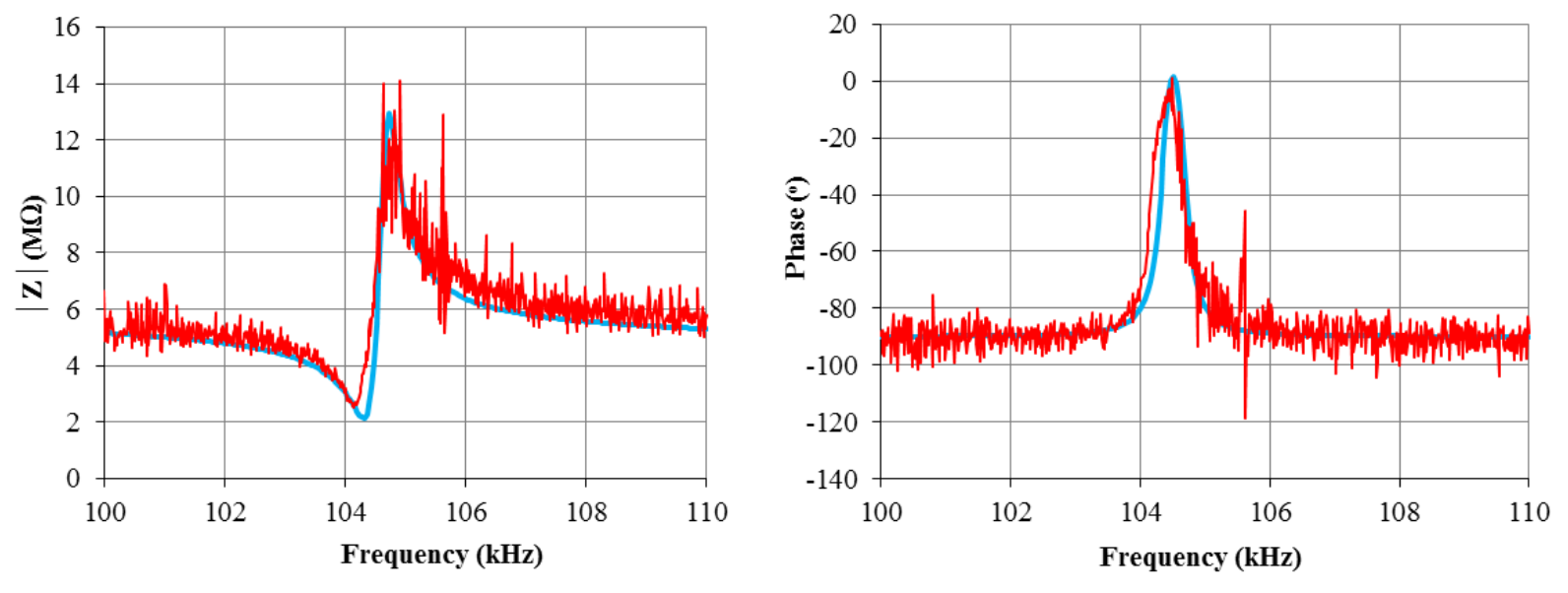

(b)
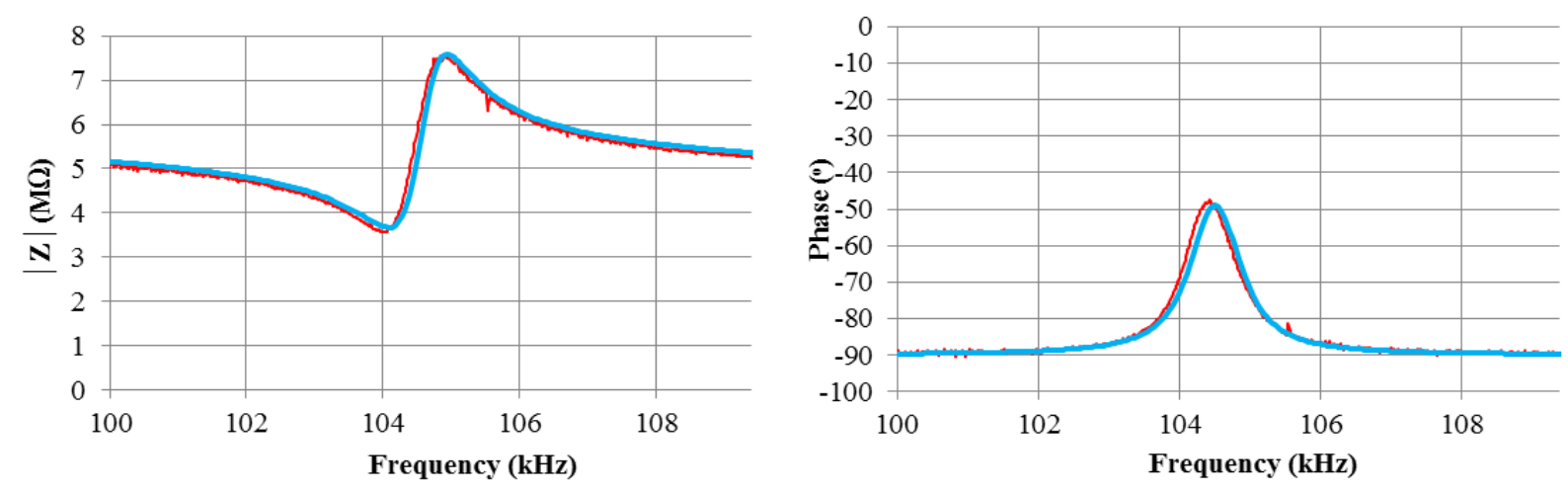

(c) 


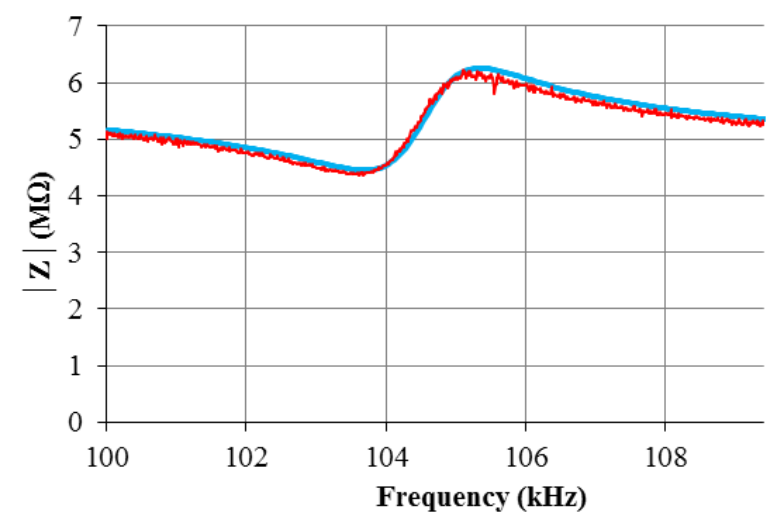

Frequency (kHz)

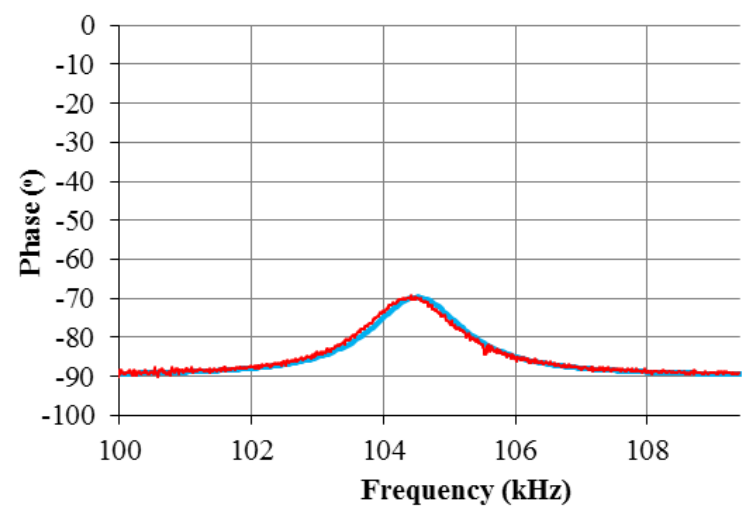

(d)

Fig. 13 Concurrence of experimental measurements with simulation results (Behavioral model) for (a) $100 \mathrm{~Pa}$ (b) $1000 \mathrm{~Pa}$ (c) $10000 \mathrm{~Pa}$ (d) $101325 \mathrm{~Pa}$

The higher noise in Fig. 13 (a) and (b) is due to the lower oscillation voltage, $60 \mathrm{mV}$ required to prevent nonlinear behavior due to excessive oscillation amplitude at low pressures, between $100 \mathrm{~Pa}$ and $1000 \mathrm{~Pa}$ while the oscillation voltage has been increased to $120 \mathrm{mV}$ for $P_{a}$ above $1000 \mathrm{~Pa}$, as shown in Fig. 13 (c) and (d) to achieve a better signalto-noise ratio.

At a near vacuum pressure of $100 \mathrm{~Pa}$, the difference in the convergence of the observed magnitude of impedance at the resonant frequency of experimental measurements and simulations is $0.25 \mathrm{M} \Omega$ with an error of $6.3 \%$ while that at the anti-resonant frequency is $0.13 \mathrm{M} \Omega$ with an error of $0.86 \%$. The difference and error in observed phase at the resonant frequency is $0.37^{\circ}$ and $2.85 \%$.

At a low pressure of $1000 \mathrm{~Pa}$, the difference between the observed impedance magnitudes at the resonant frequency is $0.1 \mathrm{M} \Omega$ with an error of $3.74 \%$ while that at the anti-resonant frequency is $0.7 \mathrm{M} \Omega$ with an error of $5.46 \%$. The difference and error in observed phase at the resonant frequency is $0.48^{\circ}$ and $3.98 \%$.

At a moderate pressure of $10000 \mathrm{~Pa}$, the difference between observed magnitudes of impedance at the resonant frequency is $0.096 \mathrm{M} \Omega$ with an error of $2.612 \%$ while that at the anti-resonant frequency is $0.005 \mathrm{M} \Omega$ with an error of $0.06 \%$. The difference and error in observed phase at the resonant frequency is $0.16^{\circ}$ and $0.32 \%$.

At atmospheric pressure, the difference between the resonant frequencies as observed in simulations and through experimental results is $0.05 \mathrm{kHz}$ while the difference in observed impedance magnitude is $0.32 \mathrm{M} \Omega$ giving an error percentage of $4.35 \%$ at the resonant frequency. While the difference observed at the anti-resonant frequencies (as a result of parasitic capacitance effects) is $0.1 \mathrm{kHz}$ while that in observed impedance magnitude is 0.007 with an error percentage of $0.14 \%$. The difference in phase at the resonant frequencies is $0.199^{\circ}$ and $0.28 \%$.

For convenience, the quality factor, $Q$ obtained from analytical modeling, finite element modeling and experimental measurements are termed $Q_{M A T L A B}, Q_{F E M}$, and $Q_{E X P}$. The results are plotted at logarithmic intervals and compared in Table 2. 
Table 2 Comparison of obtained results

\begin{tabular}{|c|c|c|c|}
\hline $\begin{array}{c}\text { Pressure } \\
(\mathbf{P a})\end{array}$ & $\begin{array}{c}Q_{\text {EXP }} \text { (Cadence/ } \\
\text { Experimental) }\end{array}$ & $Q_{F E M}$ (COMSOL) & $Q_{\text {MATLAB }}$ (MATLAB) \\
\hline \multicolumn{4}{|c|}{ Molecular -flow Regime } \\
\hline 100 & 455 & 475 & 600 \\
\hline \multicolumn{3}{|c|}{ Transition-flow Regime } \\
\hline 300 & 445 & 423 & 470 \\
\hline 1000 & 363 & 345 & 420 \\
\hline 3000 & 225 & 217 & 185 \\
\hline 10000 & 133 & 128 & 87 \\
\hline 30000 & 85 & 79 & 80 \\
\hline \multicolumn{5}{|c|}{ Slip-flow Regime } \\
\hline 40000 & 76 & 65 & 75 \\
\hline 50000 & 70 & 60 & 66 \\
\hline 60000 & 65 & 57 & 63 \\
\hline 101325 & 60 & 55 & \\
\hline
\end{tabular}

When $K_{n}$ is greater than 10 , the device behavior is analyzed in the molecular flow regime. The range of surrounding pressure is characterized to be below $272 \mathrm{~Pa}$, taking the characteristic length of the reported device as $2.5 \mu \mathrm{m}$. In this regime, the mean free path of the molecule is much greater than the characteristic length of the device. As a result, no boundary layers are formed, and the intermolecular collisions are neglected. The molecules reflected back from the surface of the device do not collide with the mean free molecules, thus neglecting the inter-molecular collisions (Pandey 2008). The structural damping alone is responsible for computing the $Q$ in this case. Since the analytical model does not employ structural damping for the device, the analytical $Q$ shoots up as other sources of damping are negligible at such low pressures. This explains the non-convergence between the analytical and experimental values of $Q$. On the other hand, the finite element model includes both, structural damping, and film damping effects.

For values of $K_{n}$ ranging between 0.1 and 10, the surrounding pressure is characterized to lie between $272 \mathrm{~Pa}$ and $27200 \mathrm{~Pa}$. Here, the behavior of the resonator is analyzed in the transition flow regime where the mean free path of the molecule is comparable to the characteristic length of the device (Pandey 2008). The interaction between the surface of the device and the molecules in the gap is comparable to the inter-molecular interaction. Hence, both structural damping and film damping play a significant role in the computation of the $Q$, making the analysis complicated. As discussed in Section 3.3.1, choosing $\mathrm{Al}$ as the membrane material helps in reducing the material losses while the use of $\mathrm{W}$ as the material for the via increases the effective mass of the device. Besides, the anchor losses are minimized by implementing the Bulk mode thus minimizing the energy loss from the system. A higher convergence of experimental and numerical values (obtained from finite element modeling) is obtained as compared to the analytical ones in this range. Thus, from the tabulated data, we can infer that the analytical model holds valid from ranges of pressure, $272 \mathrm{~Pa}$ and higher.

As values of the Knudsen number reduces to lie in the range of 0.01 and 0.1 , the range of pressure varies from 27200 $\mathrm{Pa}$ to pressures beyond atmospheric pressure. The designed structure is made to operate in the slip flow regime to enhance the device performance i.e. enhance the sensitivity. The characteristic length of the device is reduced to accomplish to target the sensitivity enhancement. In this regime, the behavior of the resonator does not follow ordinary continuum laws (Pandey 2008). Appropriate slip boundary conditions were implemented in the analytical model to account for velocity slip effects. The convergence of $Q$ obtained from the analytical, finite element modeling and experimental results in this regime are much higher than that in the other two regimes.

As discussed in the previous sections, the resonant frequency obtained for the device during experimental simulations is $103.6 \mathrm{kHz}$ that corresponds to $97.6 \mathrm{kHz}$ during three-dimensional finite element simulations in COMSOL Multiphysics at atmospheric pressure. The difference in observed resonant frequency owes to the change in material 
used for suspensions in device i.e. $\mathrm{Al}$ and $\mathrm{TiN}$ have been used to design the device suspensions while $\mathrm{Al}$ has been used for simplicity in the finite element model.

Several readings were taken to analyze the sensitivity of the reported device. The readings were taken at an interval of $300 \mathrm{~Pa}$ in the molecular flow regime, $1000 \mathrm{~Pa}$ in the transition flow regime and $10000 \mathrm{~Pa}$ in the slip flow regime. The sensitivity of the reported device was observed to be promising in the molecular flow regime and transition flow regime. When the surrounding pressure is below $30000 \mathrm{~Pa}$, the $\mathrm{Q}$ of the device reduces by an approximate factor of 70 with an increase in $100 \mathrm{~Pa}$. As the surrounding pressure increase and the behavior of the device is analyzed in the transition flow regime i.e. from a range of $300 \mathrm{~Pa}<P_{a}<30000 \mathrm{~Pa}$, the change in $Q$ is reduced by a factor of 40 to 0.1 with an increase in $100 \mathrm{~Pa}$. In the slip flow regime, where $P_{a}>30000 \mathrm{~Pa}, Q$ reduces by an approximate factor of 0.04 on an average with a rise in $100 \mathrm{~Pa}$.

As mentioned earlier (Referring to Equation (3)), $Q=\omega_{r} m / c_{\text {total }}$. The ratio of resonant frequencies has matched to a great extent with the ratio of quality factors of obtained from experimental measurements and finite element modeling that is the ratio of $\omega_{r} / c_{\text {total }}$, considering $m$ as a constant. This is done to compute the damping coefficients computed from the corresponding methodology and compare them on the same scale.

The damping coefficient for analytical, finite element modeling and experimental values were computed and compared at pressures ranging from 100 Pascal to atmospheric pressure. Table 3 shows the percentage of convergence error between the experimental measurements, finite element modeling, and analytical simulations. For convenience, the damping coefficients and resonant frequency are termed according to the corresponding methodology.

Table 3 Validation of designed model

\begin{tabular}{|c|c|c|c|c|c|c|c|}
\hline $\begin{array}{c}\text { Pressure } \\
\text { (Pa) }\end{array}$ & $\begin{array}{l}f_{r_{-} E X P} / \\
f_{r_{-} F E M}\end{array}$ & $\begin{array}{l}\boldsymbol{Q}_{E X P} / \\
\boldsymbol{Q}_{F E M}\end{array}$ & 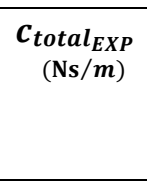 & 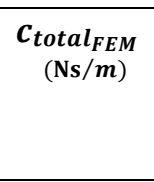 & 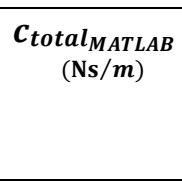 & $\begin{array}{c}\text { \% Convergence } \\
\text { Error } \\
\left(c_{\text {total }_{\text {EXP }}}^{-}\right. \\
\left.c_{\text {total }_{F E M}}\right) \\
\end{array}$ & $\begin{array}{c}\text { \% Convergence } \\
\text { Error } \\
\left(c_{\text {total }_{E X P^{-}}}\right. \\
\left.c_{\text {total }_{M A T L A B}}\right) \\
\end{array}$ \\
\hline 100 & \multirow{7}{*}{1.106} & 1.044 & $5.72 \times 10^{-7}$ & $5.16 \times 10^{-7}$ & $4.34 \times 10^{-7}$ & -10.813 & -31.868 \\
\hline 300 & & 0.951 & $5.85 \times 10^{-7}$ & $5.80 \times 10^{-7}$ & $4.54 \times 10^{-7}$ & -0.9 & -23.596 \\
\hline 1000 & & 0.95 & $7.17 \times 10^{-7}$ & $7.11 \times 10^{-7}$ & $6.20 \times 10^{-7}$ & -0.884 & -15.702 \\
\hline 3000 & & 0.964 & $1.16 \times 10^{-6}$ & $1.13 \times 10^{-6}$ & $1.07 \times 10^{-6}$ & -2.373 & -8 \\
\hline 10000 & & 0.962 & $1.96 \times 10^{-6}$ & $1.92 \times 10^{-6}$ & $1.80 \times 10^{-6}$ & -2.157 & -9.023 \\
\hline 30000 & & 0.929 & $3.06 \times 10^{-6}$ & $3.11 \times 10^{-6}$ & $2.99 \times 10^{-6}$ & 1.345 & -2.353 \\
\hline 101325 & & 0.967 & $4.34 \times 10^{-6}$ & $4.23 \times 10^{-6}$ & $4.13 \times 10^{-6}$ & -2.609 & -5 \\
\hline
\end{tabular}

As evident from the tabulated data, a higher degree of convergence is observed in the damping coefficient of experimental and finite element modeling results for the designed resonator at lower pressures than the pre-computed analytical results.

\section{Conclusion}

The performance parameters of several structures were optimized against the corresponding structural parameters. Accordingly, the an optimized square plate resonator with dimensions $140 \mu \mathrm{m}$ x $140 \mu \mathrm{m}$ x $8 \mu \mathrm{m}$ with 6 x 6 perforations along the row and column respectively and an initial air gap height of $2.5 \mu \mathrm{m}$ was manufactured in 250-nm CMOS technology. The reported device exhibits a sensitivity of -0.09 at higher pressures (atmospheric pressure) and increases to -0.3 at lower pressures of $40 \mathrm{kPa}$ in the slip flow regime. The sensitivity further increases to -25 in the transition flow regime.

The variation of the quality factor is studied over a surrounding pressure ranging from $100 \mathrm{~Pa}$ to atmospheric pressure. The Knudsen number, $K_{n}$, inversely related to the surrounding pressure and characteristic length of flow, was applied to characterize the behavior of the resonator at different pressure regimes. The characteristic length of the device was 
reduced to enhance the sensitivity at atmospheric pressure and scale the atmospheric pressure to the slip flow regime. At atmospheric pressure, squeeze film damping is accounted for the most dominant damping mechanism in the formulation of the analytical model in MATLAB. Perforations were incorporated to minimize the squeeze-film damping effects in the plate. The air gap between the two plates was reduced to increase the Knudsen number, leading to a higher sensitivity of the device. Since the resonator is targeted to operate in the slip flow regime to sense higher variations of the quality factor at atmospheric pressure, appropriate boundary conditions and effective viscosity coefficient computations were incorporated in the analytical model to account for deviations from the continuum laws. However, the analytical model fails below a pressure of $272 \mathrm{~Pa}$ as it does not compute structural damping effects in the device at such low pressures in the molecular flow regime. This limitation is overcome by modeling the structure by finite element methods in COMSOL Multiphysics.

The optimized device was manufactured in 250-nm CMOS technology. This was followed by the release of the manufactured test chip in the in-house cleanroom. The frequency response obtained from the released test chip (with an Agilent 4294A precision impedance analyzer in the in-house measurement unit) was aligned with a behavioral model in Cadence to mimic the quality factor against varying pressure conditions. The experimental $Q$ of the resonator follows a power law in the operating pressure range i.e. transition flow and slip flow regime.

A comparative study of the obtained $Q$ from experimental measurements, finite element method, and analytical model has been performed to analyze the convergence and ascertain the validity and reliability of the reported model. A higher degree of convergence between the simulated damping coefficients obtained from finite element modeling and experimental results than that of analytical modeling in MATLAB in molecular flow regime validates the design methodology that authenticates the improvement over previous work.

\section{Acknowledgment}

The authors would like to thank the anonymous reviewers for their valuable comments and suggestions to improve the quality of the paper.

The work has been supported in part by the Spanish Ministry of Science and Innovation under project TEC201127047, and European Social Fund (ESF). Saoni Banerji holds an FI scholarship funding by the Catalan government and European Social Fund (ESF).

\section{References}

Baltes H, Brand O, Fedder GK, Hierold C, Korvink JG, Tabata O (eds) (2005) CMOS-MEMS. In: Timme HJ (ed) CMOS based pressure sensors, vol 2. Wiley-VCH Verlag GmbH, Weinheim, pp 257-335

Chen WC, Fang W, Li SS (2011) A generalized CMOS-MEMS platform for micromechanical resonators monolithically integrated with circuits. J. Micromech. Microeng. 21: 065012 (15pp). doi:10.1088/0960$1317 / 21 / 6 / 065012$

Yinan L, Junbo W, Zhenyu L, Deyong C, Jian C (2015) A resonant pressure microsensor capable of self-temperature compensation. Sensors 15: 10048-10058. doi: 10.3390/s150510048

Bao M, Heng Y, Yuancheng S, Yuelin W (2003) Squeeze-film air damping of thick hole-plate. Sensors and Actuators A: Physical 108: 212-217. doi: 10.1016/S0924-4247(03)00263-2

Vermuri S (2000) Behavioral modeling of viscous damping in MEMS. M.S. Thesis Report, Carnegie Mellon University 
Pandey A.K, Pratap R, Chau F.S (2006) Analytical solution of the modified Reynolds equation for squeeze film damping in perforated MEMS structures. Sensors and Actuators A: Physical 135: 839-848. doi: 10.1016/j.sna.2006.09.006

Škvor Z (1967-1968) On acoustical resistance due to viscous losses in the air gap of electrostatic transducers Acustica 19:295-297

Kim E, Cho Y, Kim M (1999) Effect of holes and edges on the squeeze film damping of perforated micromechanical structures 12th IEEE Intern. Conf. on MEMS (MEMS'99), Orlando, FL, January 17-21, pp 296-301

Pandey AK, Pratap R (2007) A comparative study of analytical squeeze film damping models in rigid rectangular perforated MEMS structures with experimental results Microfluidics Nanofluidics 4: 205-218. doi: 10.1007/s10404007-0165-4

Darling RB, Hivick C, Jianyang X (1998) Compact analytical modeling of squeeze film damping with arbitrary venting conditions using a Green's function approach. Sensors and Actuators A: Physical 70:32-41. Doi: $10.1016 /$ S0924-4247(98)00109-5

Veijola T (2006) Analytic damping model of an MEM perforation cell. Microfluidics and Nanofluidics 2 (3): 249260. doi: 10.1007/s10404-005-0072-5

Banerji S, Madrenas J, Fernandez D (2015, April 27-30) Optimization of parameters for CMOS MEMS resonant pressure sensors. Paper presented at 2015 Symposium on Design Test Integration and Packaging of MEMS and MOEMS (France), Montpellier (pp. 107-112)

Senturia SD (2001) Microsystem Design. Kluwer Academic Publishers Norwell, MA, USA

Kaajakari (2009) Practical MEMS. Small gear Publishing, Las Vegas, Nevada

Pandey AK, Pratap R, Chau FS (2008) Effect of pressure on fluid damping in MEMS torsional resonators with flow ranging from continuum to molecular regime Experimental Mechanics 48:91-106. doi: 10.1007/s11340-007-9076-2

Basu J, Bhattacharyya TK (2011) Micromechanical resonators for radio frequency communication applications. Microsyst. Technol. 17: 1557-1580. doi: 10.1007/s00542-011-1332-9

Rawat U, Pasula VV, Nair DR, Dasgupta A (2013) Efficient anchor design for quality factor enhancement in a silicon nitride-on-siliconlateral bulk mode resonator. http://www.comsol.es/paper/download/182757/rawat_presentation.pdf. Accessed 18 September 2015

Khine L (2010) Performance parameters of micromechanical resonators. Ph.D. dissertation, National University of Singapore

Brotz J (2004) Damping in CMOS-MEMS resonators. Master's Project Report, Carnegie Mellon University

COMSOL Multiphysics (2008) MEMS Module. https://extras.csc.fi/math/comsol/3.5/doc/mems/memsmodlib.pdf. Accessed 10 December 2014

Fernández D, Ricart J, Madrenas J (2010) Experiments on the release of CMOS-Micromachined metal layers. Journal of Sensors vol. 2010, Article ID 937301, 7 pages, 2010. doi:10.1155/2010/937301

Michalik P, Sánchez-Chiva J M., Fernández D, Madrenas J (2015) CMOS BEOL-embedded z-axis accelerometer Electronics Letters, 51(11), 2015, 865-867 
Kaajakari (2009) Practical MEMS. Small gear Publishing, Las Vegas, Nevada Younis MI (2011) MEMS Linear and Nonlinear Statics and Dynamics. New York, USA 


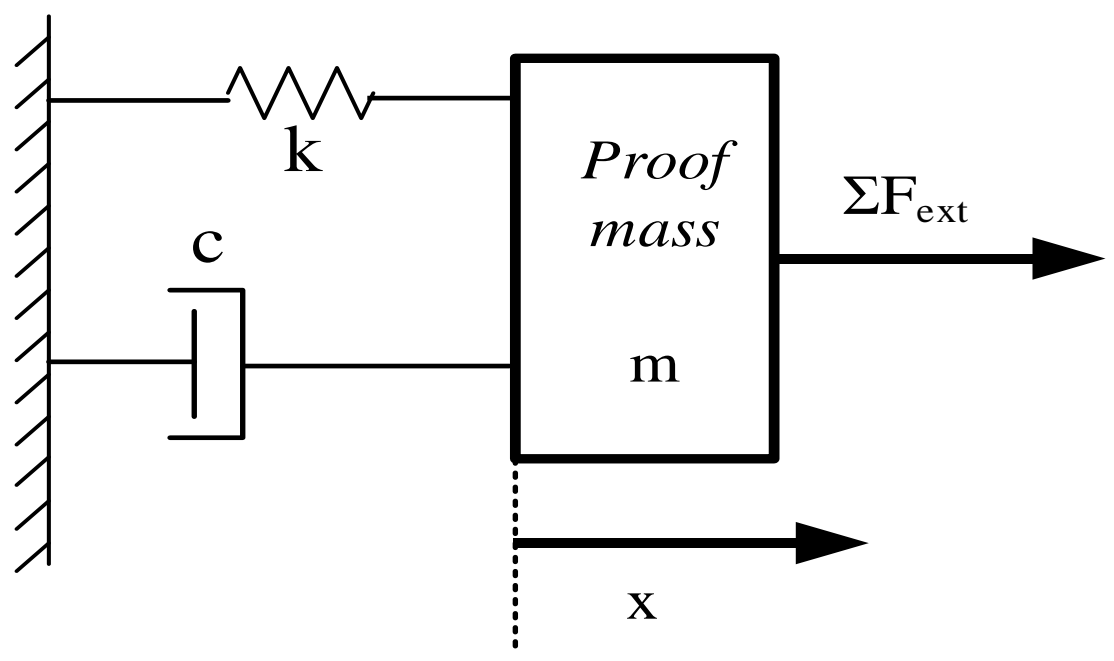

Fig. 1 Schematic of mass resonator

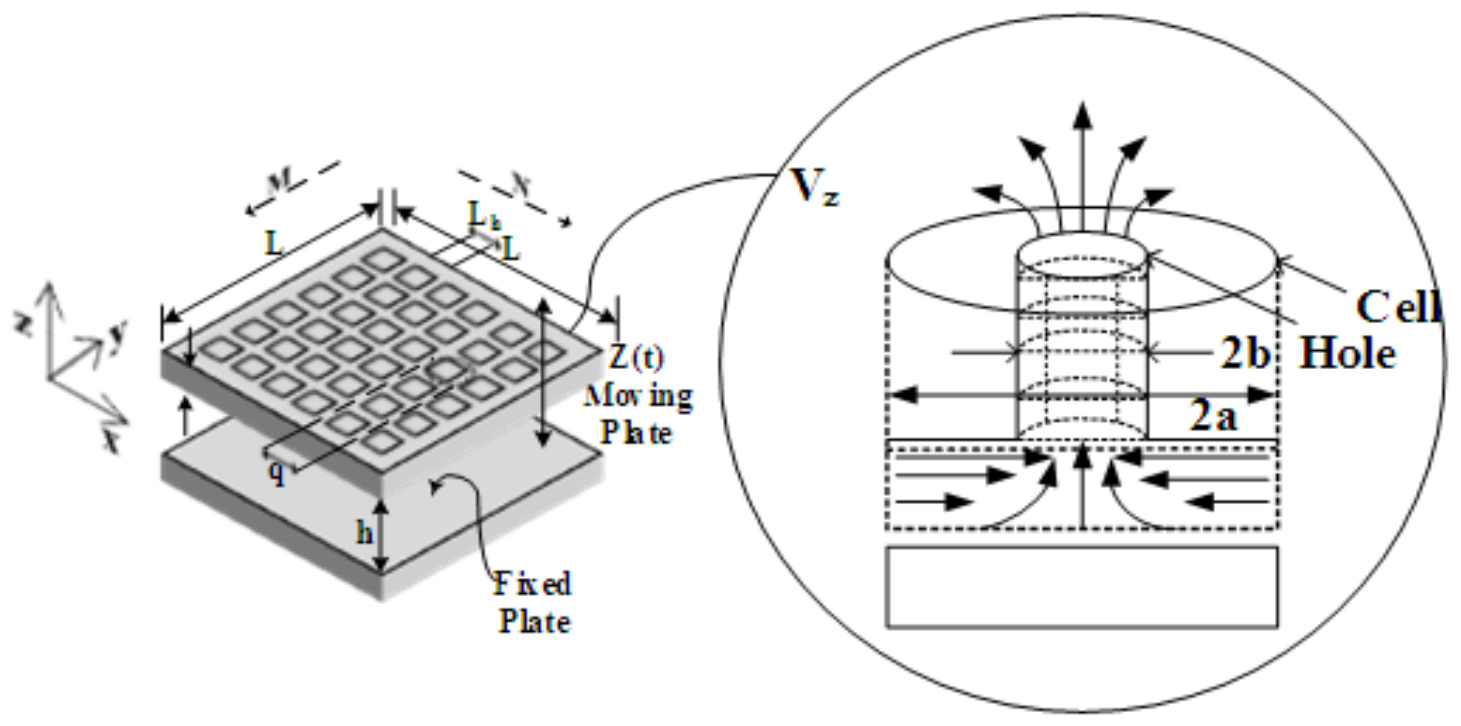

(a)

(b)

Fig. 2 Schematic diagram for squeeze-film damping in the perforated system under consideration: (a) Oscillating perforated plate and the fixed plate in isometric view

(b) Airflow dynamics under a typical perforation-cell combination (Adapted from [Pandey 2007]) 


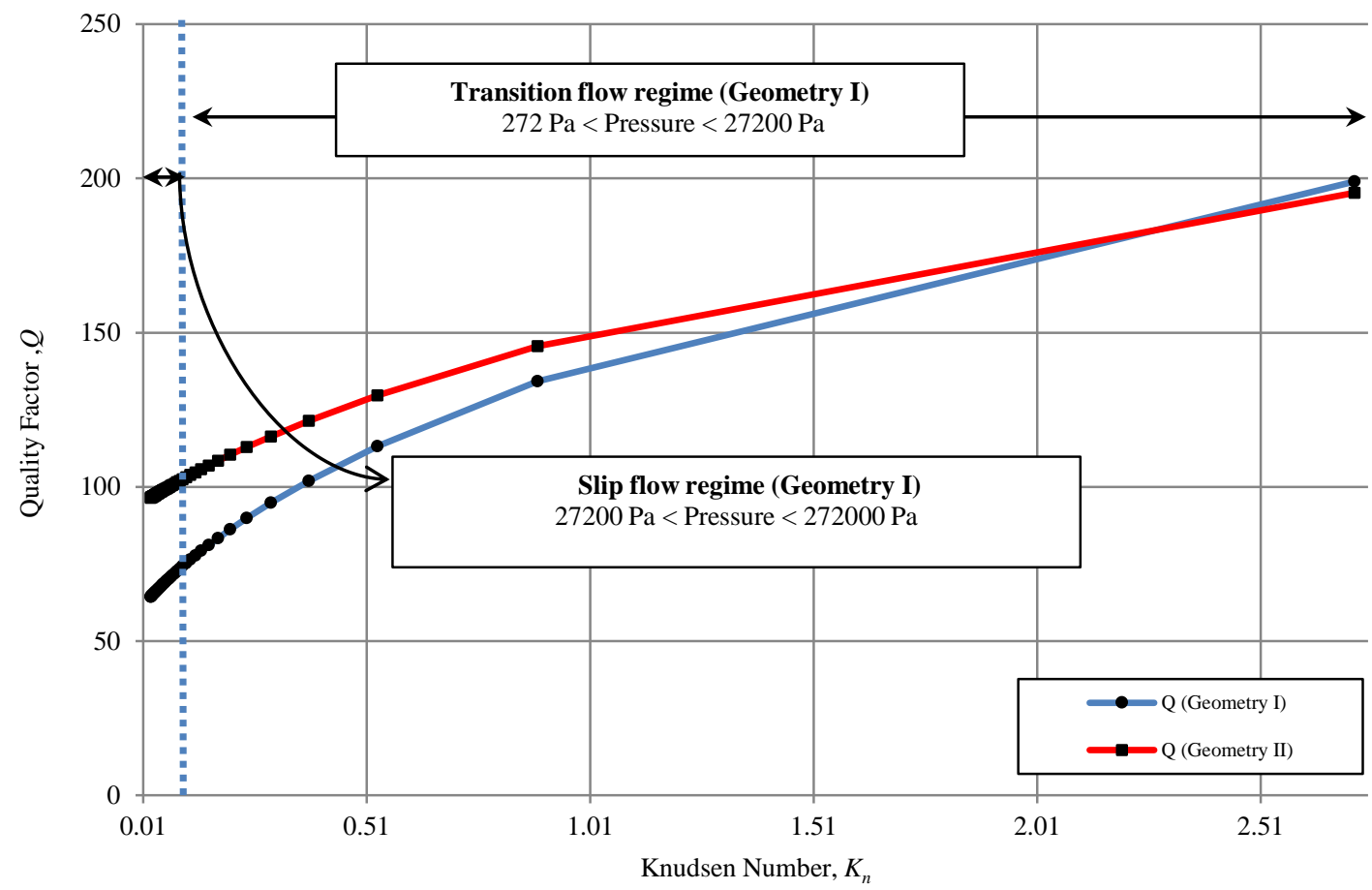

Fig. 3 Variation of the quality factor $(Q)$ with Knudsen number $\left(K_{n}\right)$ for the two geometries

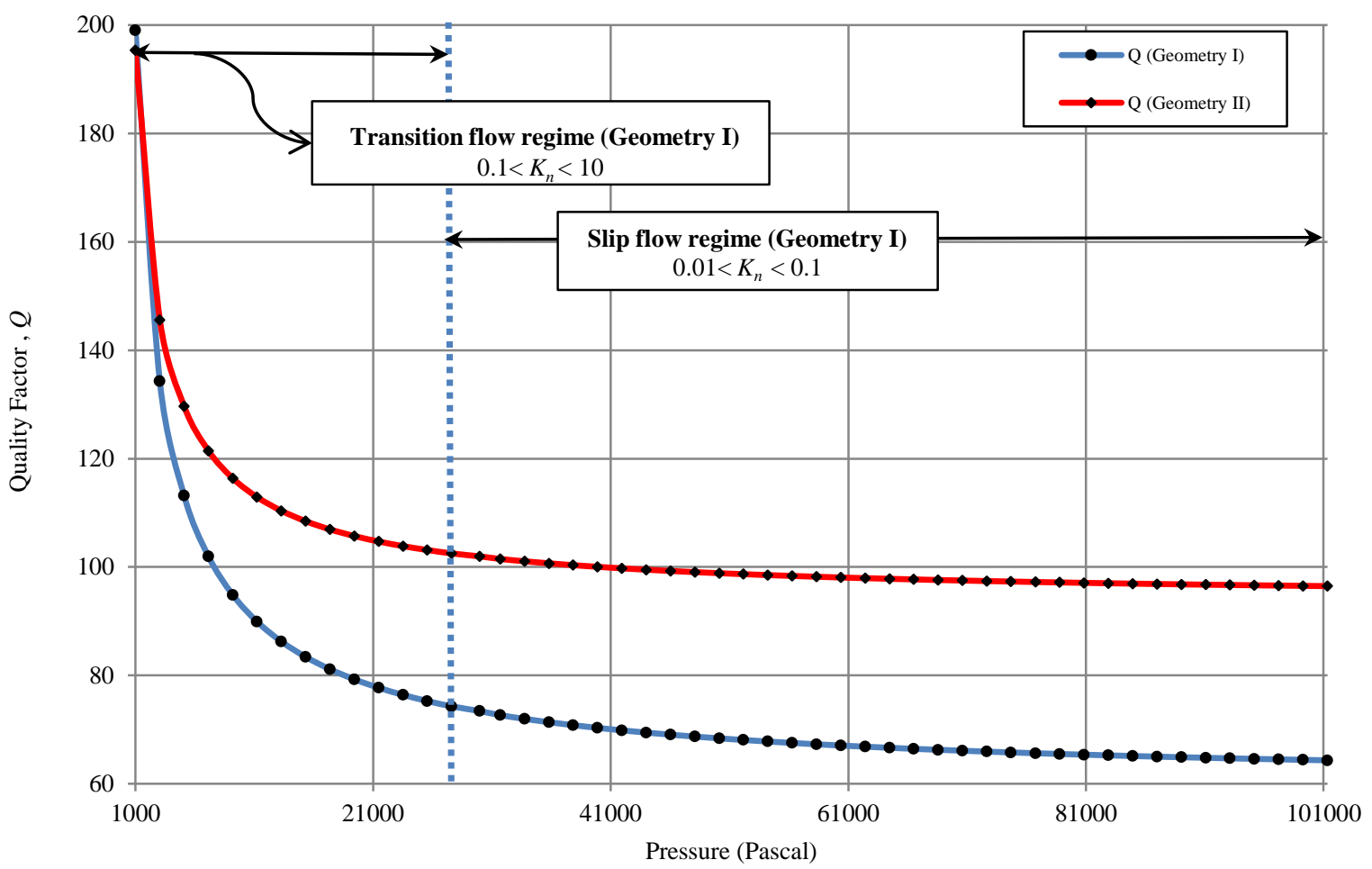

Fig. 4 Variation of the quality factor $(Q)$ with pressure $\left(P_{a}\right)$ for the two geometries 


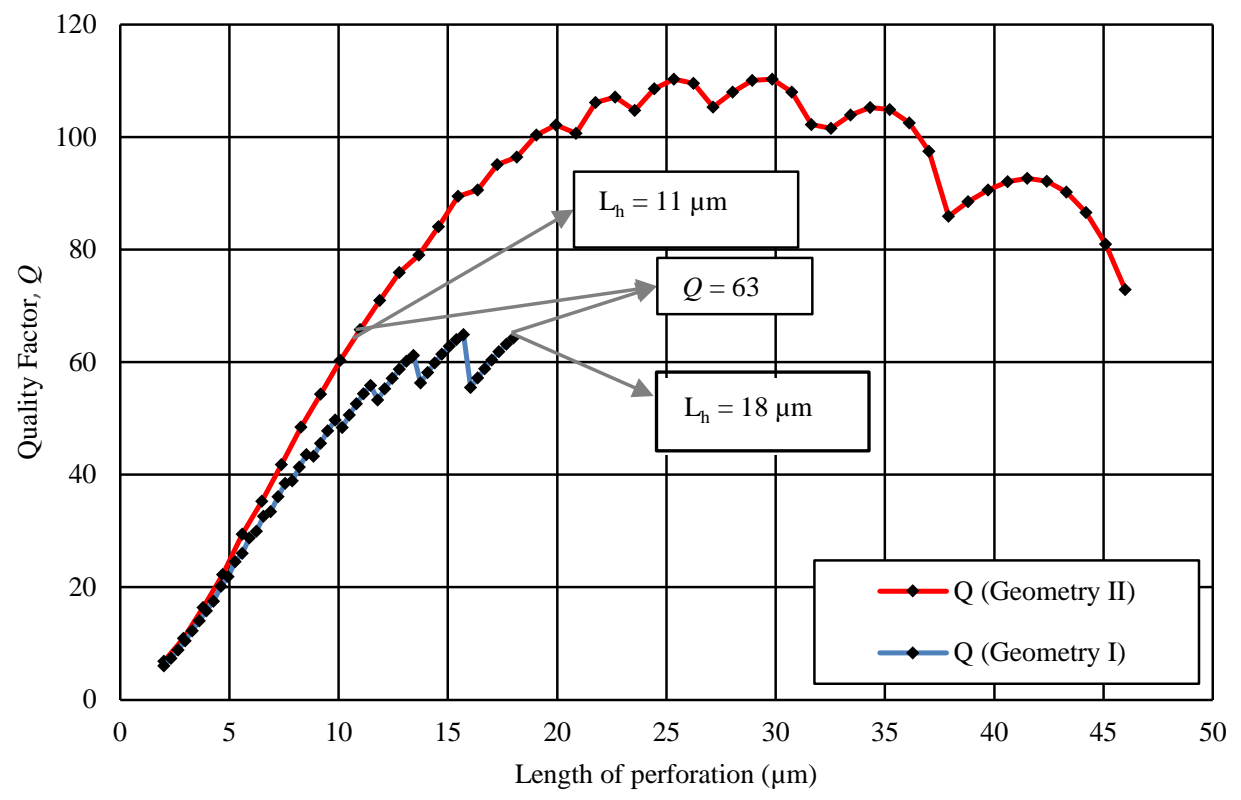

Fig. 5 Variation of quality factor (Q) vs. length of perforation $\left(L_{h}\right)$ for the two geometries

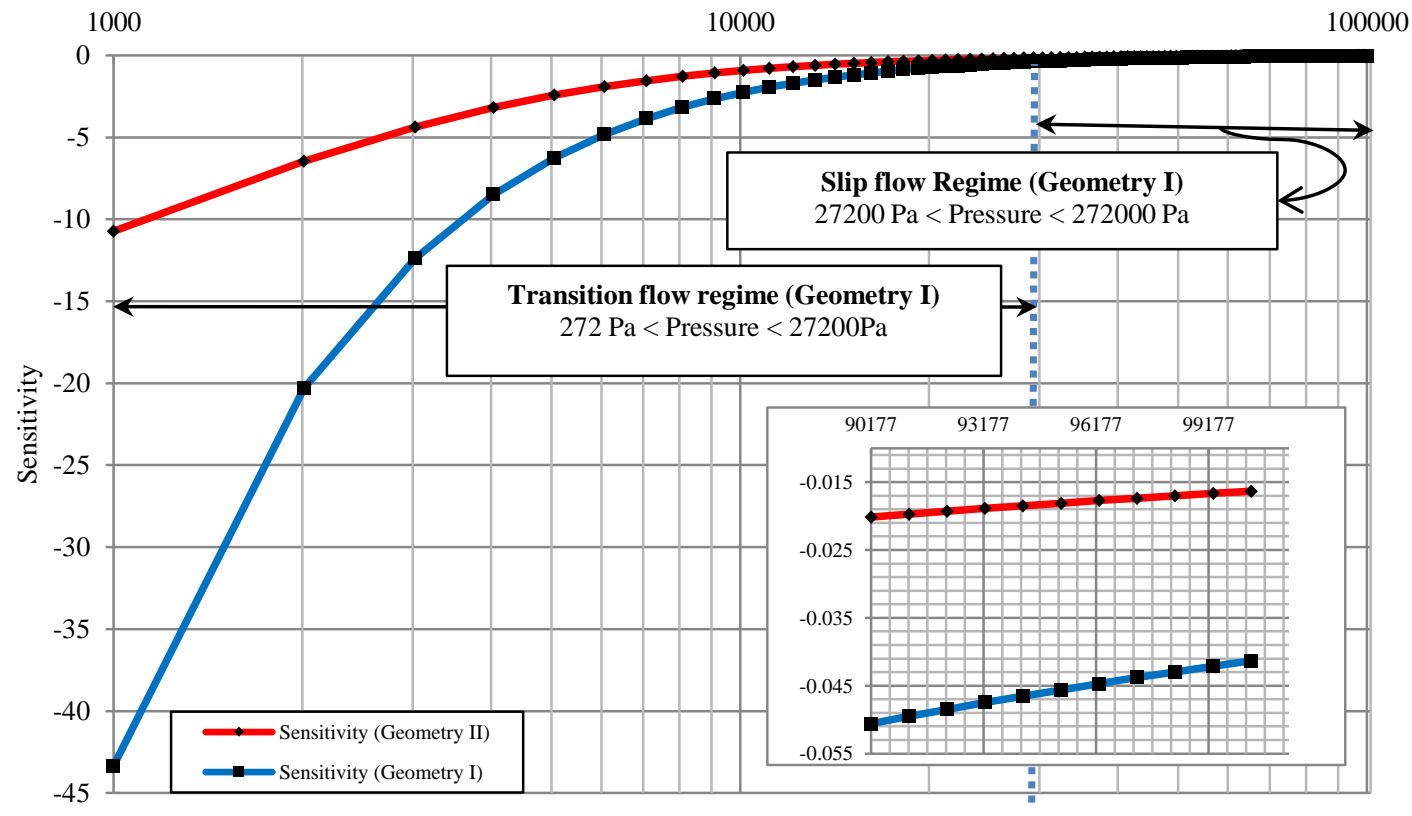

Pressure (Pascal)

Fig. 6 Sensitivity of sensor for the two geometries 


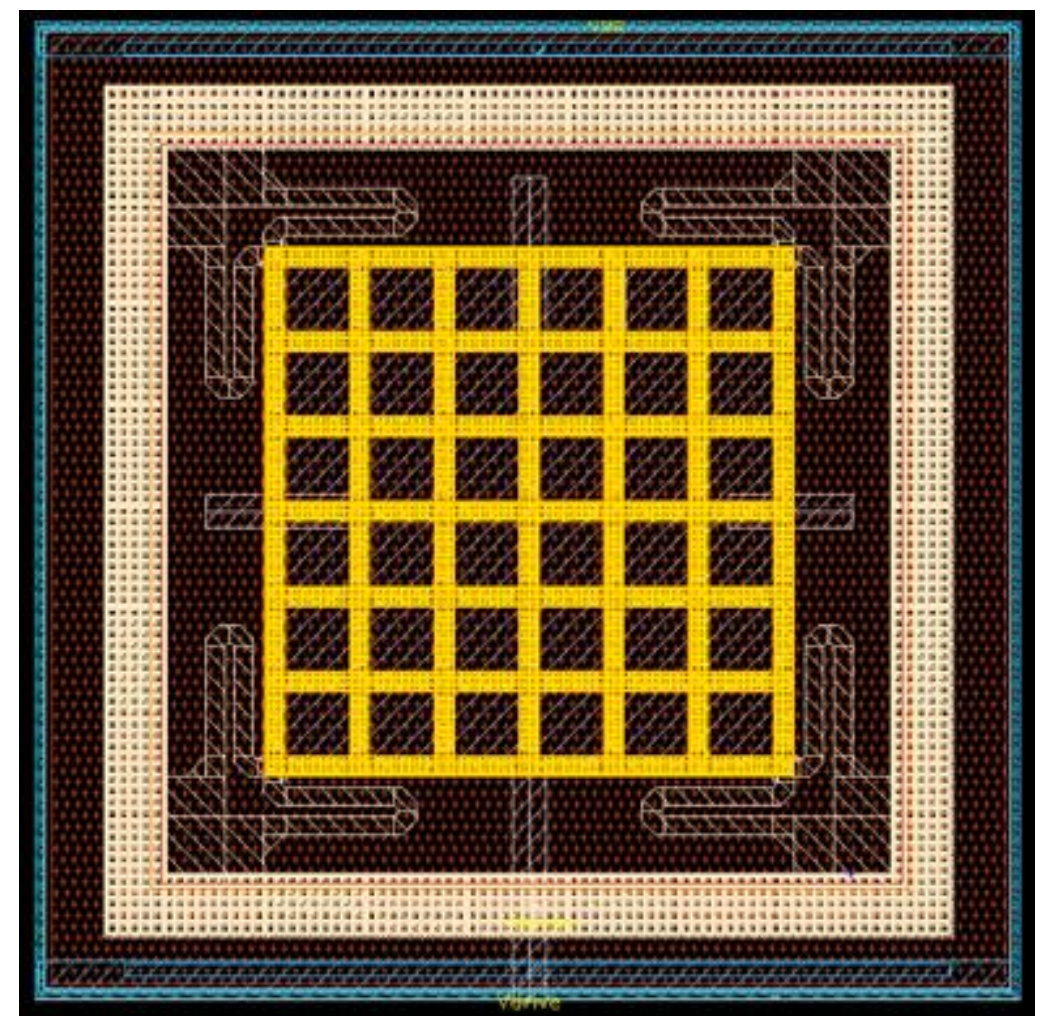

Fig. 7 Layout of optimized resonant pressure sensor

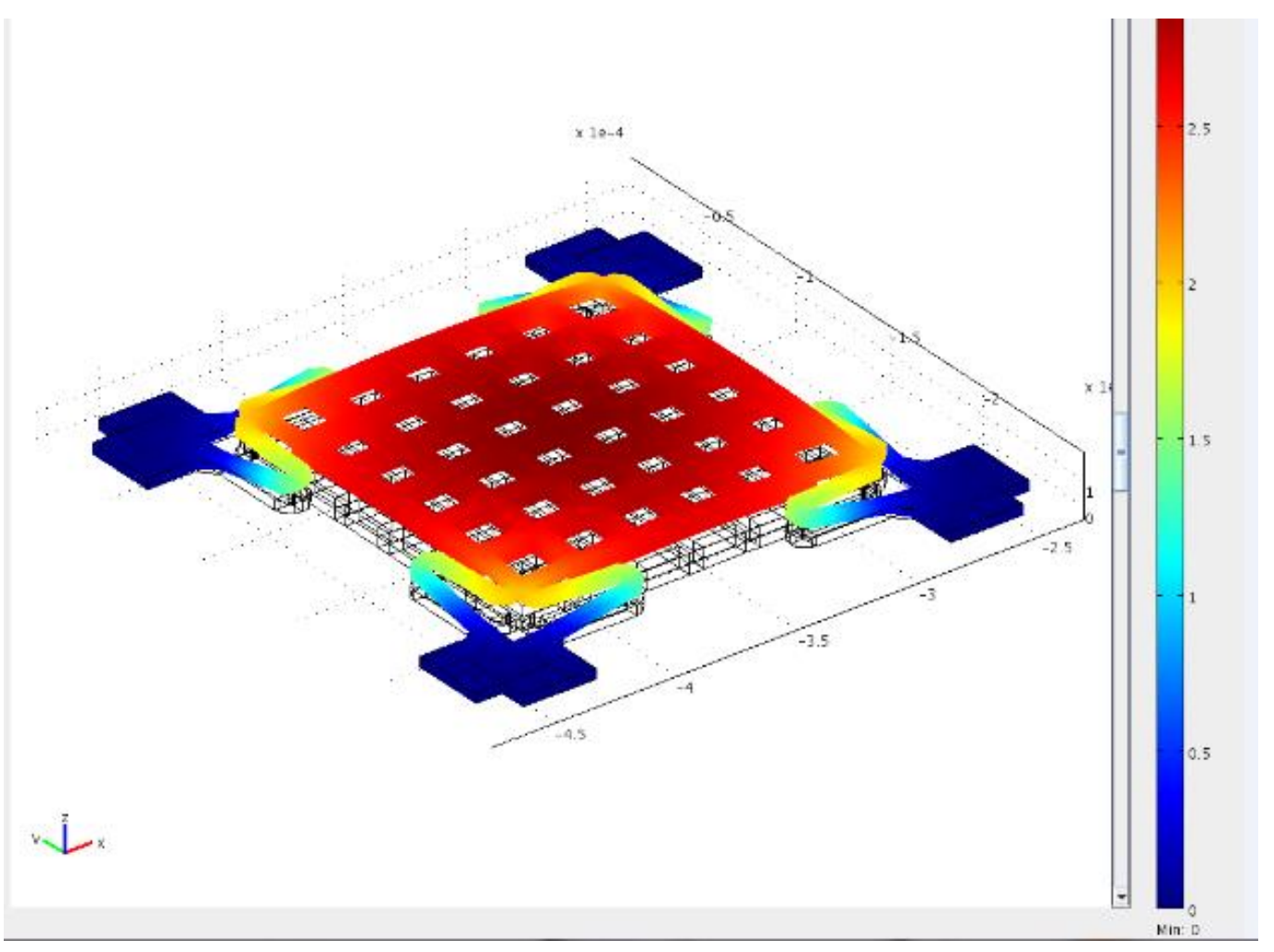

Fig. 8 Effect of damping force on designed resonant pressure sensor in COMSOL Multiphysics 


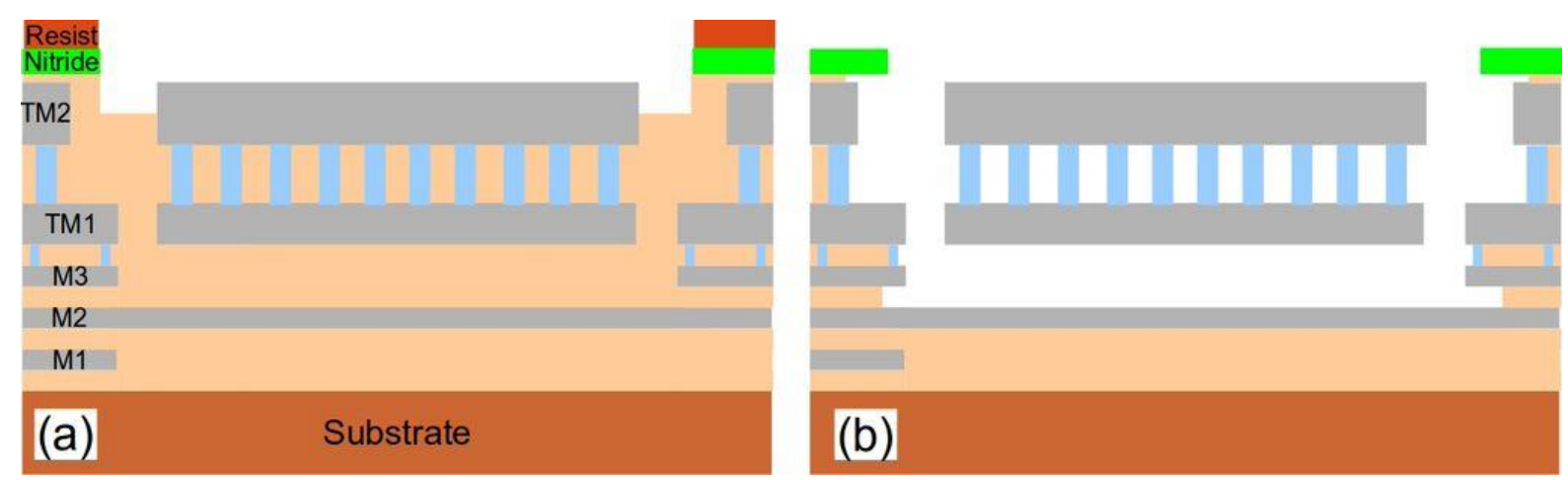

Fig. 9 Stack diagram of simplified IHP SG25H1/H3 BEOL stack diagram after wet isotropic etching till substrate (a) Pre-Release cross section (b) Post-Release cross section

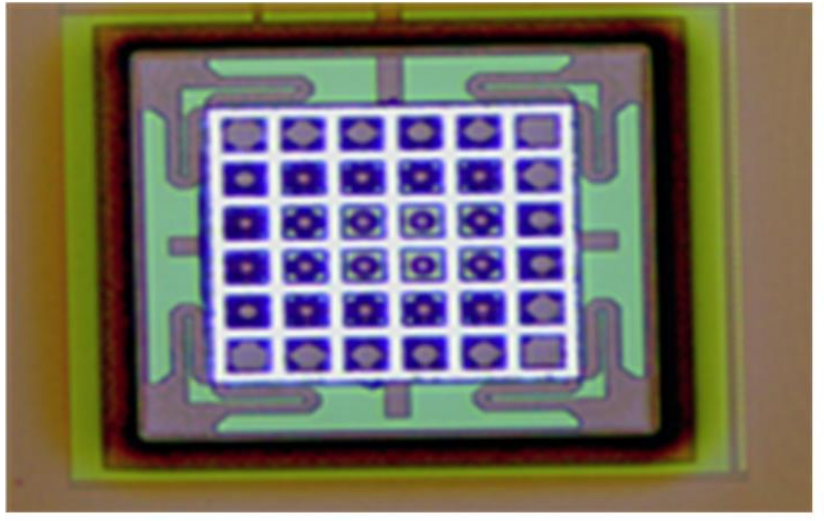

(a)

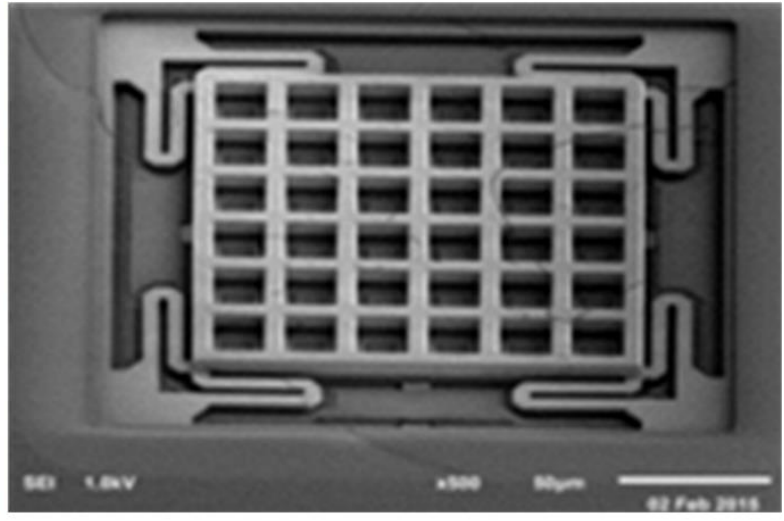

(b)

Fig. 10 View of the test Chip

(a) Microphotograph (b) Under scanning electron microscope
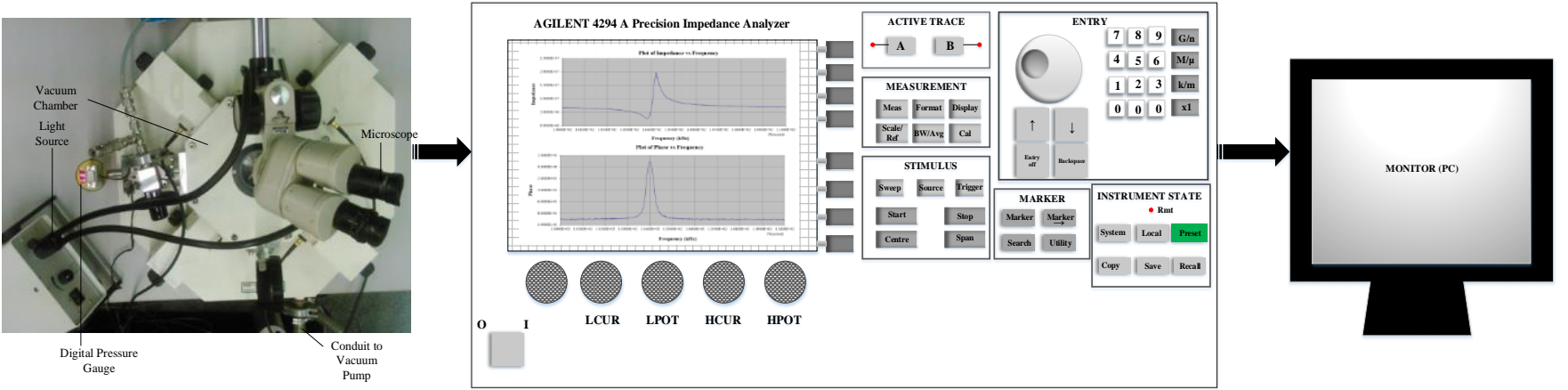

Fig. 11 Experimental set-up for obtaining frequency response from designed sensor 


\begin{tabular}{|lll|}
\hline O & Experimental Data & Curve fitting \\
\hline
\end{tabular}

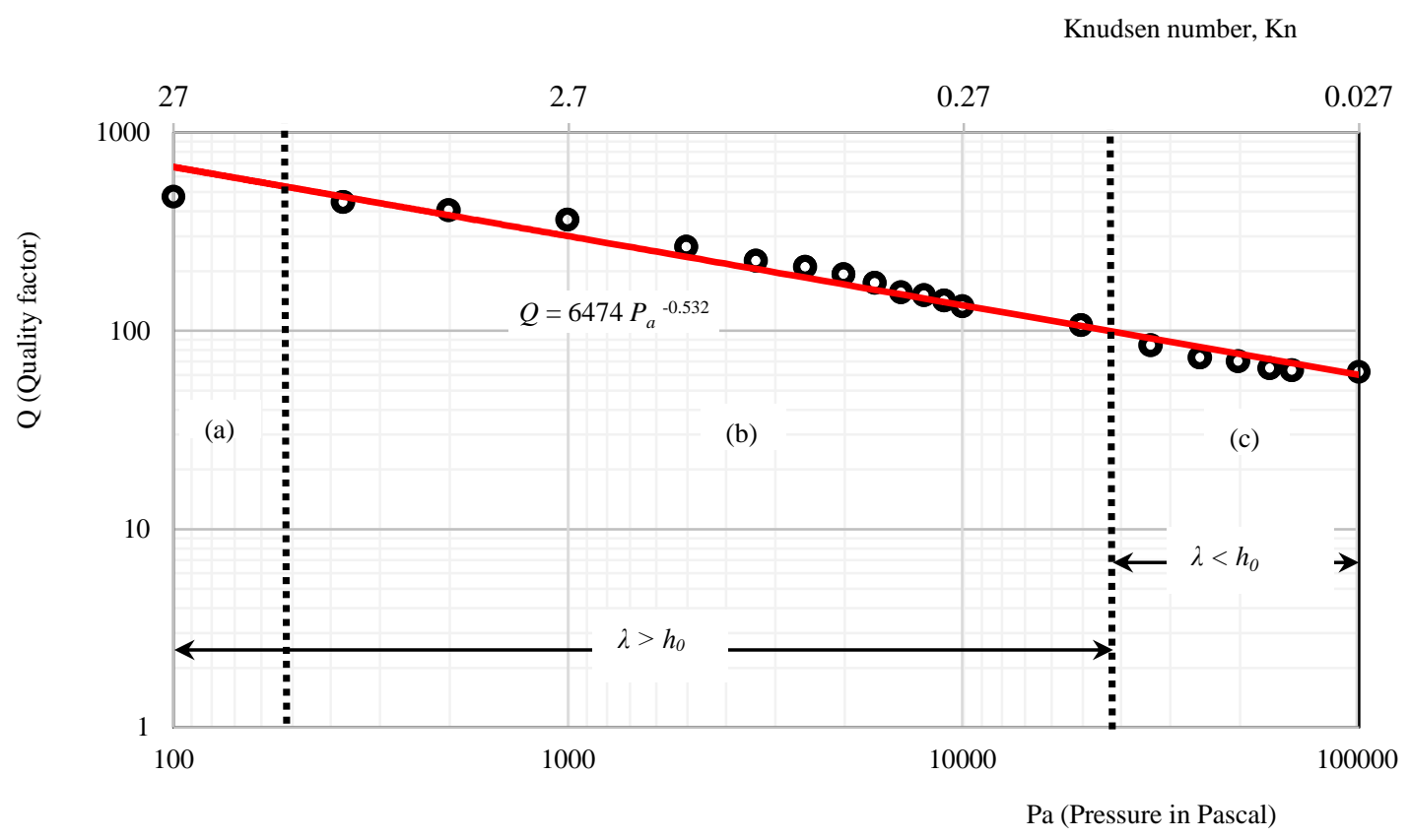

Fig. 12 Fitting of power law over (a) Molecular flow regime (b) Transition flow regime (c) Slip flow regime
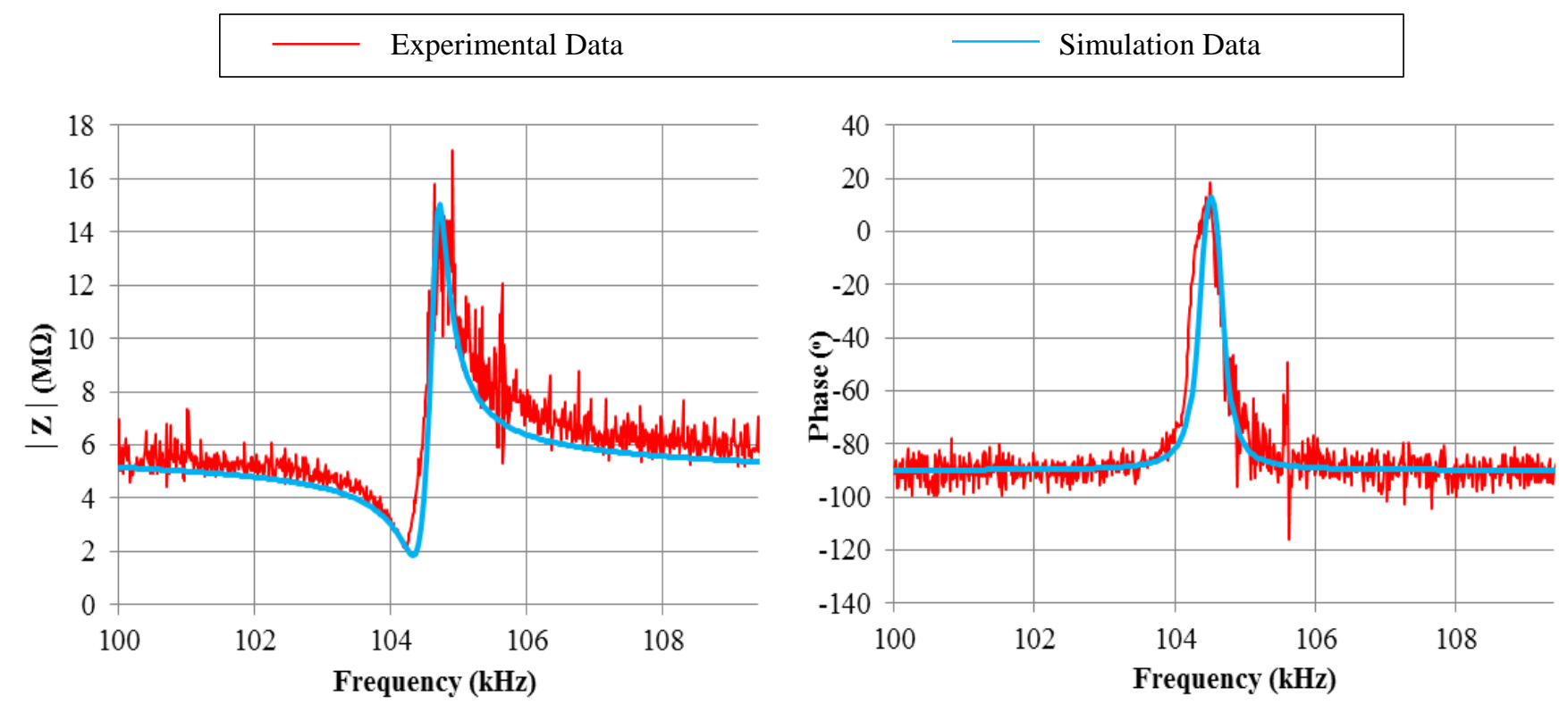

(a) 

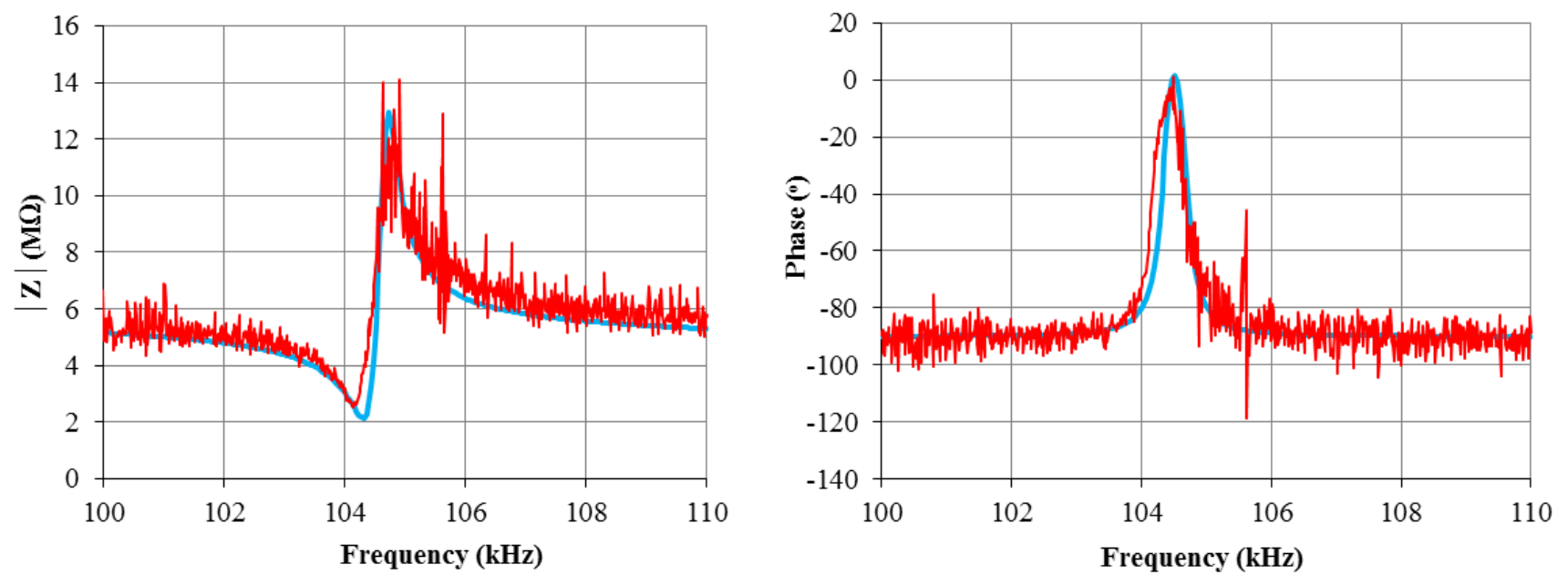

(b)
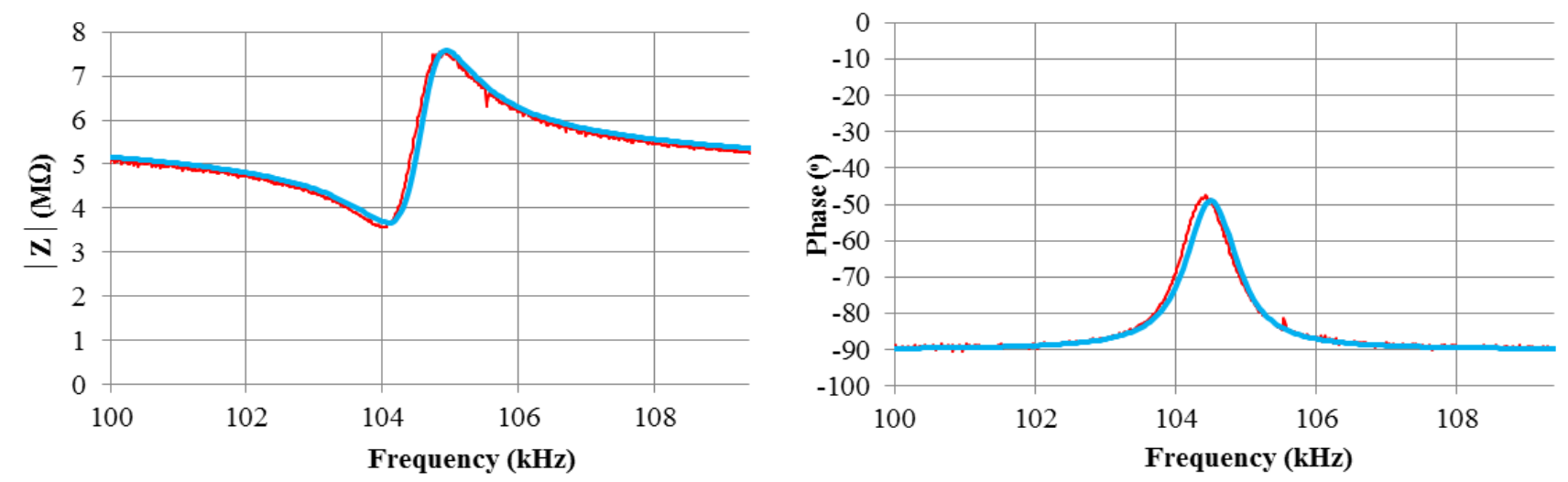

(c)
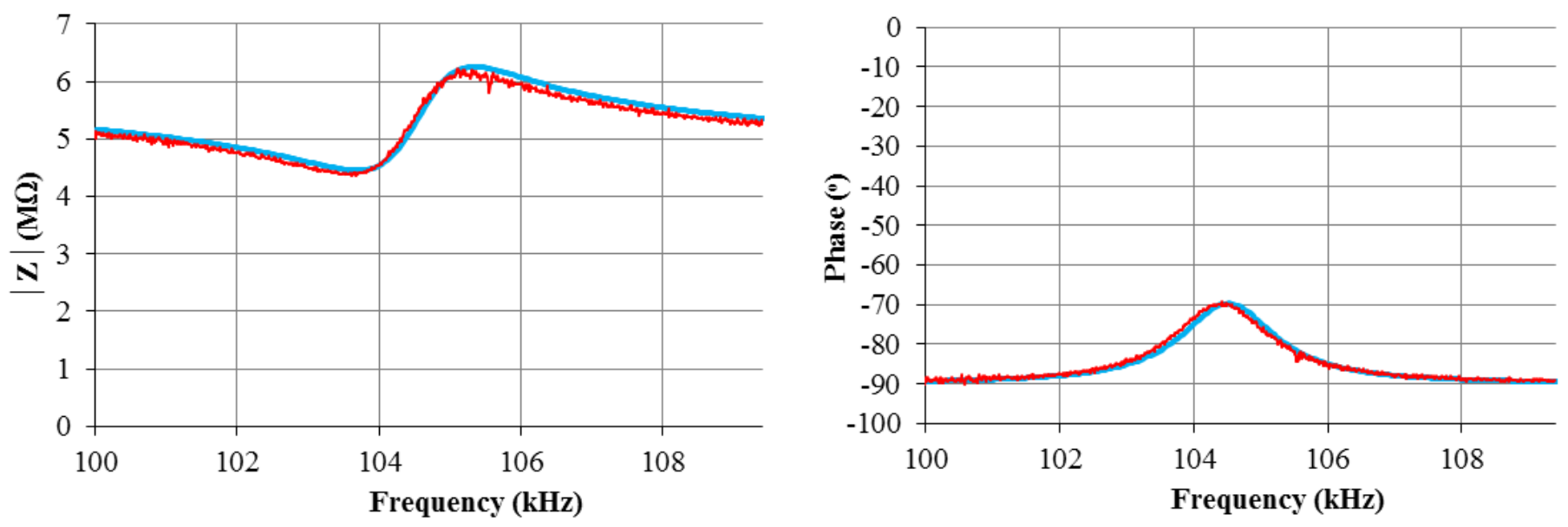

(d)

Fig. 13 Concurrence of experimental measurements with simulation results (Behavioral model) for (a) $100 \mathrm{~Pa}$ (b) $1000 \mathrm{~Pa}$ (c) $10000 \mathrm{~Pa}$ (d) $101325 \mathrm{~Pa}$ 
Table 1 Comparison of parametrization and dynamics of two geometries at standard atmospheric pressure

\begin{tabular}{|c|c|c|c|c|}
\hline \multirow[b]{2}{*}{ Symbol } & \multirow[b]{2}{*}{ Description } & \multicolumn{2}{|c|}{ Values } & \multirow[b]{2}{*}{ Units } \\
\hline & & Geometry I & Geometry II & \\
\hline \multicolumn{5}{|c|}{ Independent variables } \\
\hline$L$ & Length of the square plate & $140 \times 10^{-6}$ & $250 \times 10^{-6}$ & $\mathrm{~m}$ \\
\hline $\boldsymbol{W}$ & Width of the square plate & $140 \times 10^{-6}$ & $250 \times 10^{-6}$ & $\mathrm{~m}$ \\
\hline$T_{p}$ & Thickness of the square plate & \multicolumn{2}{|c|}{$8 \times 10^{-6}$} & $\mathrm{~m}$ \\
\hline$L_{1}$ & Length of perforation & \multicolumn{2}{|c|}{$18 \times 10^{-6}$} & $\mathrm{~m}$ \\
\hline$N_{h}(N \times M)$ & Number of perforations & $36(6 \times 6)$ & $121(11 \times 11)$ & - \\
\hline$h_{0}$ & Air gap thickness & $2.5 \times 10^{-6}$ & $4 \times 10^{-6}$ & $\mathrm{~m}$ \\
\hline$\rho_{\text {plate }}(A l-W-A l)$ & Effective density of plate & \multicolumn{2}{|c|}{6500} & $\mathrm{~kg} / \mathrm{m}^{3}$ \\
\hline$S$ & Spacing between two perforations & \multicolumn{2}{|c|}{$4 \times 10^{-6}$} & $\mathrm{~m}$ \\
\hline$\mu$ & Dynamic viscosity coefficient of air & \multicolumn{2}{|c|}{$1.67 \times 10^{-5}$} & $\mathrm{~Pa}-\mathrm{s}$ \\
\hline$\omega_{r}$ & Angular frequency of oscillation & \multicolumn{2}{|c|}{$103.6 \times 10^{3}$} & $\mathrm{~Hz}$ \\
\hline$\varepsilon$ & Vacuum permittivity & \multicolumn{2}{|c|}{$8.854 \times 10^{-12}$} & $\mathrm{~F} / \mathrm{m}^{2}$ \\
\hline$\varepsilon_{r}$ & Relative permittivity of air & \multicolumn{2}{|c|}{1} & - \\
\hline$A_{e}$ & Area of the plate & $7.936 \times 10^{-9}$ & $23.3 \times 10^{-9}$ & $\mathrm{~m}^{2}$ \\
\hline$\lambda$ & Mean free path of gas molecules & & & $\mathrm{m}$ \\
\hline \multicolumn{5}{|c|}{ Dependent Variables } \\
\hline$m$ & Effective mass & $4.127 \times 10^{-10}$ & $1.21 \times 10^{-9}$ & $\mathrm{~kg}$ \\
\hline$k$ & Spring stiffness constant & 174.86 & 513.29 & $\mathrm{~N} / \mathrm{m}$ \\
\hline$Q$ & Quality factor & 63 & 96.5 & - \\
\hline $\boldsymbol{K}_{n}$ & Knudsen number & 0.0268 & 0.0168 & - \\
\hline Cap & Capacitance & $28.11 \times 10^{-15}$ & $51.57 \times 10^{-15}$ & Farad \\
\hline$S$ & Sensitivity & -0.0413 & -0.0164 & - \\
\hline
\end{tabular}


Table 2 Comparison of obtained results

\begin{tabular}{|c|c|c|c|}
\hline $\begin{array}{c}\text { Pressure } \\
\text { (Pa) }\end{array}$ & $\begin{array}{l}Q_{E X P}(\text { Cadence/ } \\
\text { Experimental })\end{array}$ & $Q_{F E M}($ COMSOL $)$ & $Q_{\text {MATLAB }}$ (MATLAB) \\
\hline \multicolumn{4}{|c|}{ Molecular -flow Regime } \\
\hline 100 & 455 & 475 & 600 \\
\hline \multicolumn{4}{|c|}{ Transition-flow Regime } \\
\hline 300 & 445 & 423 & 470 \\
\hline 1000 & 363 & 345 & 420 \\
\hline 3000 & 225 & 217 & 185 \\
\hline 10000 & 133 & 128 & 145 \\
\hline 30000 & 85 & 79 & 87 \\
\hline \multicolumn{4}{|c|}{ Slip-flow Regime } \\
\hline 40000 & 76 & 65 & 80 \\
\hline 50000 & 70 & 60 & 75 \\
\hline 60000 & 65 & 57 & 66 \\
\hline 101325 & 60 & 55 & 63 \\
\hline
\end{tabular}

Table 3 Validation of designed model

\begin{tabular}{|c|c|c|c|c|c|c|c|}
\hline $\begin{array}{l}\text { Pressure } \\
\text { (Pa) }\end{array}$ & $\begin{array}{l}f_{r_{-} E X P} / \\
f_{r_{-} F E M}\end{array}$ & $\begin{array}{l}Q_{E X P} / \\
Q_{F E M}\end{array}$ & $\begin{array}{c}\boldsymbol{c}_{\text {total }_{\text {EXP }}} \\
(\mathrm{Ns} / \boldsymbol{m})\end{array}$ & $\begin{array}{c}\boldsymbol{c}_{\text {total }} \\
\quad(\mathrm{Ns} / \boldsymbol{m})\end{array}$ & $\begin{array}{c}\boldsymbol{c}_{\text {total }_{\text {MATLAB }}} \\
(\mathrm{Ns} / \mathrm{m})\end{array}$ & $\begin{array}{c}\text { \% Convergence } \\
\text { Error } \\
\left(c_{\text {total }_{E X P}}-\right. \\
\left.c_{\text {total }_{F E M}}\right)\end{array}$ & $\begin{array}{c}\text { \% Convergence } \\
\text { Error } \\
\left(c_{\text {total }_{E X P}}\right. \\
\left.c_{\text {total }_{M A T L A B}}\right)\end{array}$ \\
\hline 100 & \multirow{7}{*}{1.106} & 1.044 & $5.72 \times 10^{-7}$ & $5.16 \times 10^{-7}$ & $4.34 \times 10^{-7}$ & -10.813 & -31.868 \\
\hline 300 & & 0.951 & $5.85 \times 10^{-7}$ & $5.80 \times 10^{-7}$ & $4.54 \times 10^{-7}$ & -0.9 & -23.596 \\
\hline 1000 & & 0.95 & $7.17 \times 10^{-7}$ & $7.11 \times 10^{-7}$ & $6.20 \times 10^{-7}$ & -0.884 & -15.702 \\
\hline 3000 & & 0.964 & $1.16 \times 10^{-6}$ & $1.13 \times 10^{-6}$ & $1.07 \times 10^{-6}$ & -2.373 & -8 \\
\hline 10000 & & 0.962 & $1.96 \times 10^{-6}$ & $1.92 \times 10^{-6}$ & $1.80 \times 10^{-6}$ & -2.157 & -9.023 \\
\hline 30000 & & 0.929 & $3.06 \times 10^{-6}$ & $3.11 \times 10^{-6}$ & $2.99 \times 10^{-6}$ & 1.345 & -2.353 \\
\hline 101325 & & 0.967 & $4.34 \times 10^{-6}$ & $4.23 \times 10^{-6}$ & $4.13 \times 10^{-6}$ & -2.609 & -5 \\
\hline
\end{tabular}


Click here to access/download Attachment to Manuscript Title_Page_MITE.docx 